Elsevier required licence: (C) <2021>. This manuscript version is made available under the CC-BY-NCND 4.0 license http://creativecommons.org/licenses/by-nc-nd/4.0/

The definitive publisher version is available online at

[https://www.journals.elsevier.com/journal-of-sound-and-vibration] 


\section{Journal of Sound and Vibration \\ A comparison of time and frequency domain-based approaches to laser Doppler vibrometer instrument vibration correction \\ --Manuscript Draft--}




1. Revised frequency domain based LDV base vibration correction technique presented.

2. Enhanced signal processing shown to yield factor of seven performance improvement.

3. Recently proposed time domain processing method performance rigorously examined.

4. Model developed to relate correction signal synchronisation error and performance.

5. Model validated against experimental data with excellent agreement.

6. Framework defined for selection of correction technique and hardware specification. 


\section{Detailed Response to Reviewers}

Hello,

We would like to express our gratitude for accepting the paper. The references have been amended, however, if any issues remain please let us know. Additionally, in this manuscript, the credit author statement was removed and now is only in the Word file attached. I hope this isn't an issue. Thank you.

Kind regards,

The authors 
1

2

3

\title{
A comparison of time and frequency domain-based approaches to laser Doppler vibrometer instrument vibration correction
}

\author{
Abdel Darwish ${ }^{\mathrm{a}, *}$, Ben Halkon ${ }^{\mathrm{a}, \mathrm{b}}$, Steve Rothberg ${ }^{\mathrm{c}}$, \\ Sebastian Oberst ${ }^{\mathrm{b}}$, Robert Fitch ${ }^{\mathrm{a}}$ \\ ${ }^{a}$ School of Mechanical and Mechatronic Engineering, Faculty of Engineering $\mathcal{E}$ IT, \\ University of Technology Sydney, Ultimo, NSW 2007, Australia \\ ${ }^{b}$ Centre for Audio, Acoustics and Vibration, Faculty of Engineering \& IT, \\ University of Technology Sydney, Ultimo, NSW 2007, Australia \\ ${ }^{c}$ Wolfson School of Mechanical, Electrical and Manufacturing Engineering, \\ Loughborough University, Loughborough, Leicestershire, LE11 3TU, United Kingdom
}

\begin{abstract}
When laser Doppler vibrometers are used in the presence of ambient vibration, it is essential to compensate for the additional vibration signal content. In practice, compensation is realised by independently determining the instrument vibration and subtracting it from the erroneous measurement. When these vibrations are transient in nature, time domain-based processing should be used to carry out the correction. However, recent implementation of such an approach on stationary signals showed a factor of eight increase in performance over the previously established frequency domain-based alternative. Therefore, the work described in this paper initially focuses on determining the cause of the inconsistency and proposes a revised frequency domain approach. This revised approach offers near-equivalent performance to its time domain-based equivalent, with the latter approach offering only a factor of 0.26 increase in performance. However, despite the advantages of selecting the time domain-based technique, it typically requires high oversampling factors to allow for the accurate synchronisation of the various transducer type signals. Up until now, the only method available to deter-
\end{abstract}

\footnotetext{
${ }^{*}$ Corresponding Author

Email address: abdel.darwish@student.uts.edu.au (Abdel Darwish)
} 
mine the relationship between the sampling frequency and the performance would be experimentally, which is laborious and time consuming. Therefore, the significance of this paper is the development and experimental validation of an analytical model which predicts the sampling frequency dependence of the time domain correction technique performance. Using this, a framework was developed which allows for the optimal implementation of either correction technique and specifies the required acquisition parameters.

Keywords: mobile laser Doppler vibrometry, vibration measurement, non-stationary instrument vibration correction, time domain signal processing, transient vibration

\section{Introduction}

Laser Doppler vibrometers (LDVs) have become indispensable and widely adopted vibration measurement tools [1]. Increasingly they have been applied to mobile applications, which include buried landmine detection [2-5], terrestrial seismology [6], orbital seismology [7-9], and vibratory health assessment from drones [10]. The integration of LDVs into autonomous vehicles, such as unmanned aerial vehicles (UAVs), is a recent addition to the extensive and ever-growing plethora of LDV applications which has been receiving increased attention in recent years $[11,12]$. Such solutions unlock enormous potential for truly autonomous and remote measurement campaigns within traditionally inaccessible, remote or hazardous environments. There has, however, been a particular recent fundamental advance that has enabled the pursuit of this ambitious application domain.

Specifically, this advance relates to correction of the measured signal, where the contribution of the LDV's own vibration - otherwise indistinguishable from the intended measurement - can be completely removed, thereby fully recovering the target surface vibration velocity. Establishing solutions for LDV measurement correction has been completed for: single beam devices in the presence of arbitrary, six degree-of-freedom vibration [13]; scenarios in which beam steering optics, which might vibrate independently of the sensor head, are used [14]; and more recently, for scanning LDVs, where the laser beam scan angle must also be accounted for [15].

Dual beam LDVs can be configured to optically subtract the motion of 
the sensor head by mixing the measurement beam from the target with a reference beam from a static surface. However, this correction technique has two main practical limitations in the context of mobile LDV applications. Previous work has shown that to obtain full six degree-of-freedom correction the correction measurement must occur along the beam axis [11]. This would require the reference beam to be colinear and pointing in the opposite direction to the measurement beam and focused on the static reference surface. While this might be realisable in the laboratory, for mobile, field-based applications, this is practically impossible to achieve due to the simultaneous positioning and focusing requirements. Conversely, accelerometers are robust, readily available and enable direct measurement of sensor head vibration. Recently developed solutions have, therefore, been focused on their use, thereby offering accessible options for the practising vibration engineer.

In general, independent vibration measurements of the additional velocity contributions to the LDV signal are required and these are obtained using specifically positioned accelerometers. These additional velocity contributions can be due to a combination of sensor head, scanning head and steering optic vibration; for the sake of brevity, all solutions will be referred to as LDV measurement correction. Depending upon the geometry of the particular set-up, components of these measurements are used to compensate for the additional velocity in the direction of the laser beam. Post-processing has been conducted in the frequency domain where the necessary integration of the accelerometer signals for velocity and their synchronisation with the LDV signal is conveniently implemented. Furthermore, frequency domain representation is well-understood and is commonplace within typical vibration measurement and structural dynamic characterisation campaigns where signals are stationary in nature.

In more real-world relevant, field-based vibration measurement scenarios, including those involving the integration of LDVs with autonomous vehicles $[11,12]$, it can be reasonably expected, however, that both the target and the instrument vibration signals will be transient in nature. For this reason, an alternative approach based entirely in the time domain, was recently proposed and its performance for stationary signals compared against the established frequency domain-based equivalent [16]. While both techniques offer a significant improvement in the corrected LDV signal, the time domainbased technique outperformed its counterpart by a factor of approximately 
eight. The performance difference outcome was unexpected, especially considering that previous work has shown frequency domain integration to be the most accurate [17]. This paper will, therefore, explore the reasons for this performance gap before proposing a revised frequency domain-based approach with significantly improved performance.

In either domain, the quality of the measurement correction is sensitive to signal synchronisation since any error therein will adversely affect the quality of the corrected velocity estimate. Therefore, the development of a model which relates synchronisation error to the velocity estimate error is paramount. Firstly, the time delay estimate will always have an associated uncertainty, however small; this is minimised using a rigorous relative calibration procedure. Secondly, in the time domain, the implementation of the synchronisation is constrained to integer multiples of the time step, therefore, even a perfect delay estimate is unlikely to lead to perfect synchronisation. While interpolation could be used to upsample time domain data to enable sub-time step alignment, this is not always desirable. Therefore, a thorough investigation into the relationship between the time step and the synchronisation error is required such that an optimal sampling frequency can be selected, maximising the performance of the time domain-based technique.

The model is validated using significantly oversampled experimental data, downsampled to simulate acquisition over a range of sampling frequencies. This method of experimentally obtaining the sampling frequency dependence also enables the comparison of the time and frequency domain-based techniques across an extended range. Specifically, comparing the relative performances leads to the definition of distinct regions, within each of which the measurement correction outcome can be optimised by selecting the appropriate technique. These findings are then generalised, based on two parameters, to determine the minimum sampling frequency necessary for the time domain-based technique to outperform both others. This in turn enables the user to define the optimal hardware characteristics required for a given measurement campaign. This can be crucial when integrating such sensor solutions into autonomous vehicles as payload versus performance is in a delicate balance. 


\section{Overview of the correction measurement setup}

Practically, the correction of LDV measurements in the presence of sensor head vibration involves the use of properly positioned accelerometers to obtain the correction measurements. The number and positioning of these correction accelerometers is determined by the specific nature of the optical setup. For a single-beam LDV, a single accelerometer mounted to the rear of the sensor head colinear with the beam axis is required [11]. This relatively simple setup is convenient for the development of new processing techniques. However, the techniques developed here could be easily expanded to more complex setups which require multiple correction accelerometers, such as a scanning LDV [15].

Fig. 1 illustrates the experimental setup used in this work. It is common with that used in previous work $[11,16]$ and allows for the independent control of both the target and the LDV vibration. Here, the target vibration is the measurement of interest, while the base vibration simulates the effects of instrument vibration on the LDV measurement. Both the target and the base vibrations were realised using electrodynamic shakers independently driven using uncorrelated broadband white noise up to $200 \mathrm{~Hz}$, generated by a Siemens Digital Industries Software Simcenter SCADAS Mobile data acquisition system and accordingly amplified. The base shaker was a Tira Vibration exciter S 51120 amplified by a Tira Vibration BAA 500 and the target shaker was a Brüel \& Kjær V201 M4-CE amplified by a Brüel \& Kjær LDS LPA100. While a flat shaker/amplifier response over the frequency range of interest may be desirable, it is not essential since the correction algorithm is completely effective, irrespective of level and phase, across the entire frequency range. 


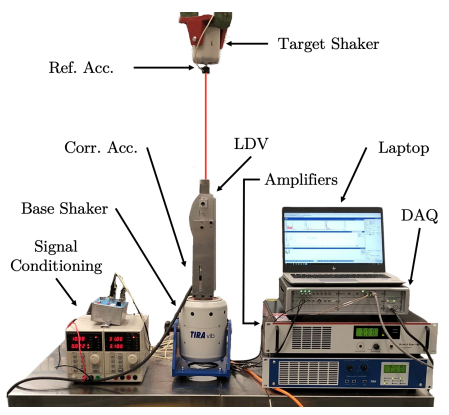

(a) General schematic of the setup used for this work. (b) Physical setup with the laser beam highlighted.

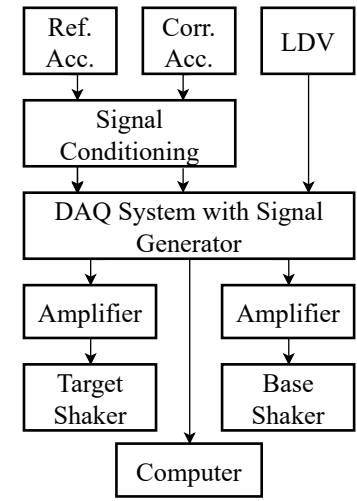

(c) Block diagram representation.

Figure 1: Experimental setup used to simulate a LDV target vibration measurement during base motion vibration. The labels "Corr. Acc." and "Ref. Acc." represent the correction and reference accelerometers, respectively.

A custom-made aluminium mounting bracket was used to fix a Polytec NLV-2500-5 Compact Laser Vibrometer to the base motion shaker so that the laser beam axis was aligned with that of the vibration. An Endevco 770F10-U-120 (200 mV/g nominal) DC-response accelerometer was mounted to the bracket with its sensitive axis colinear with that of the LDV. The target shaker was suspended directly above the LDV from an overhead crane, providing isolation from the large base motion shaker. A second Endevco accelerometer of the same model was mounted to the spigot providing the 'true' vibration measurement. As in earlier work [11], a second, fixed LDV could equally be used for the true vibration measurement. However, one or both beams would need to be off-axis to enable optical access and this may require the angular misalignment to be determined and accounted for. In this work, the use of the reference accelerometer was therefore preferred.

There are some practical limitations with the use of accelerometers, including the flatness of their amplitude and phase response. Unlike LDVs, accelerometer performance is typically limited by the first mechanical resonance of the mass-spring system. However, they are relatively low cost, are readily available and can offer acceptable performance in the context of mobile LDV measurement campaigns which are the focus of the solutions developed in this body of work. In general, a frequency range from several $\mathrm{Hz}$ to several hundred $\mathrm{Hz}$ is considered appropriate with vibration levels on the order of several tenths to several tens of $\mathrm{mm} / \mathrm{s}$. Over such a relatively narrow frequency range, it is appropriate to compensate for the amplitude 
and phase response with a straightforward relative calibration (to the LDV) and this will be described in detail subsequently.

Another limitation of accelerometers is that they typically exhibit a small amount of transverse sensitivity which might degrade correction performance in the presence of significant off-axis vibration, in this case it is only $3 \%$ [18]. In the experimental setup used here, inevitable rocking motion of the shakers is minimised by centring the mass distribution on the shaker axis and this effect is therefore considered to be negligible. Nevertheless, this and some misalignment between the shaker axes also results in some motion of the LDV beam on the target. While this motion was insufficient to cause the laser beam to deviate substantially from the region of interest on the target, pseudo-vibrations in the LDV signal, which include speckle noise, are associated with such relative motion of the laser beam across the target surface and these cannot be corrected by the means proposed in this paper. However, combined LDV sensitivity to transverse vibration as a result of both phenomena is on the order of $0.1 \%$ [19] and is therefore also considered to be negligible in the context of sensitivity to sensor head vibration [11].

\section{An improved frequency domain-based processing approach}

Accepting the LDV measurement as the reference, the accelerometer sensitivities must be adjusted and the signals synchronised prior to being used in post-processing. Both of these require that the accelerometer signals are integrated, however, the integration of a discretised signal commonly leads to the introduction of errors which can manifest themselves as drift. Drift is more readily identified and relatively easily removed in the time domain by subtracting a first order least squares fit. It is, however, less noticeable in the frequency domain and is practically difficult to remove. Conversely, integration is readily implemented and more accurate in the frequency domain [17]. An improved approach to both the relative calibration procedure and the LDV measurement correction is achieved by implementing a combination of time and frequency domain processing techniques.

\subsection{Accelerometer signal relative sensitivity and time delay}

Relative sensitivity determination and time delay estimation first require that a vibration measurement is obtained from all transducers with their 
sensitive axes aligned. Practically, this was achieved using an arrangement with the LDV positioned directly above and focused on an accelerometer stack in turn mounted to the spigot of a shaker. Care was taken to eliminate contamination from ambient vibration by placing the entire arrangement on an anti-vibration base.

The vibration signals are measured and processed according to the procedure shown in Fig. 2 for a single accelerometer channel. In earlier work $[11,13,15]$, signals were directly captured as frequency spectra, calculated from Hann-windowed time blocks because the excitation was broadband white noise. Following frequency domain integration of the accelerometer signals, Sensitivity Prefactor and Temporal Alignment values were determined as per the ultimate step in the diagram. In the revised approach, time data are instead acquired with the accelerometer signal immediately converted to frequency domain representation, albeit without prior application of a Hann window to the time data blocks. While perhaps considered unconventional, the lack of windowing is an essential part of the technique as it enables preservation of the overall time domain waveform, thereby allowing more accurate subsequent detrending.

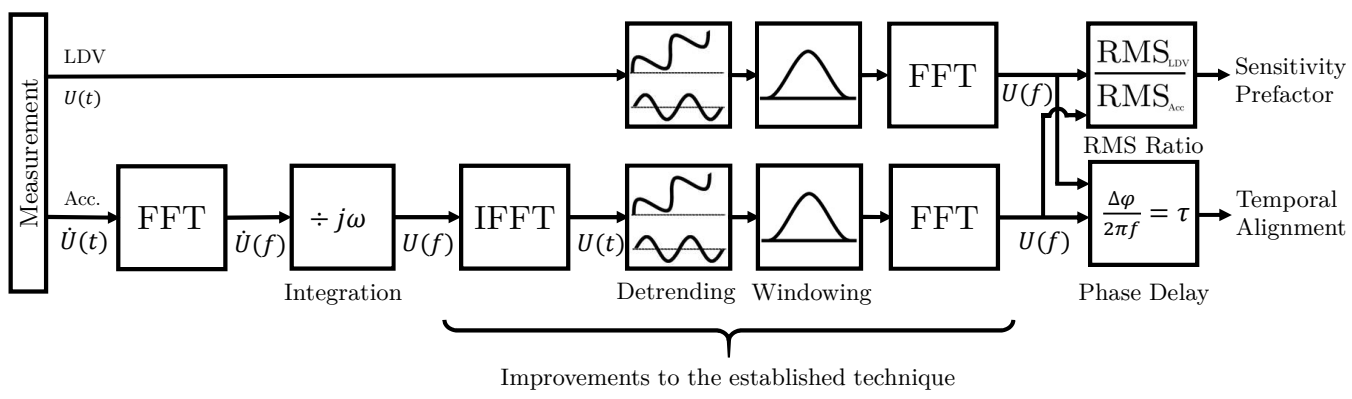

Figure 2: A schematic of the new frequency domain-based relative calibration procedure. The improvements are highlighted by the curly brackets and include the addition of the IFFT, detrending and FFT stages, along with moving the Windowing stage from after the measurement block to just before the second FFT. The signal "Acc." represents that obtained from either the correction or reference accelerometer.

As can been seen in Fig. 2, a $j \omega$ division is used in the frequency domain to integrate the accelerometer signal. Removal of the resulting drift is achieved by the subtraction of a first order least squares fit from the time domain integrated signal. Since this detrending step will act to remove not 
only the spurious but also some genuine signal content, the same operation must be applied to the measured LDV signal. Both signals are now converted to the frequency domain in the usual way, and implementing a Hann window on the time data blocks if required. The required Sensitivity Prefactor and Temporal Alignment parameters are obtained by taking the ratio of the Root Mean Square (RMS) values and from the phase difference between the signals, respectively.

Fig. 3a shows phase difference plots generated from a single time data block using the established and the improved frequency domain-based method. By comparing the two curves, it becomes obvious that detrending leads to increased agreement between the two types of transducers. As can be seen in Fig. 3b, the improvement occurs mainly occurs at the lower frequencies. For a system with a constant phase delay, the group delay can be written as:

$$
\Delta \phi=-2 \pi f \tau_{\text {meas }}
$$

where $\Delta \phi$ is the phase difference, $\tau_{\text {meas }}$ is the measured time delay and $f$ is the frequency. Therefore, a least squares fit can be used to extract $\tau_{\text {meas }}$ from the detrended data set. For this dataset, a value of $\tau_{\text {meas }}=-133.3 \pm 1.8$ us was obtained and is consistent with equivalent values previously observed for such sensors and signal conditioning.

\subsection{Instrument vibration correction}

Correction of the LDV measurement similarly requires integration of the correction accelerometer signals with detrending therefore being essential for optimal performance. A revised post-processing approach is shown in Fig. 4. As for the relative sensitivity adjustment and time delay calculation process, the differences between this improved and the previously established approach are largely captured in the steps to the right of the IFFT and to the left of the second FFT. Again, the signals are now captured in the time domain whereas previously frequency spectra were captured directly. In this case, the integration-related steps are conducted on both the correction and target reference accelerometers, these having had their relative sensitivities adjusted and signal time delays estimated. The latter of the two accelerometers is only intended for use in the laboratory research campaign where which provides a 'true' vibration measurement for correction performance; for subsequent real-world campaigns, there is no such device since, otherwise, there 


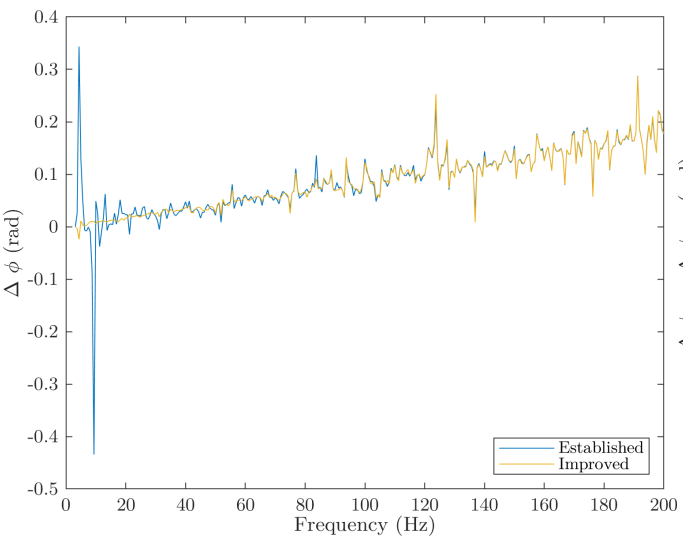

(a)

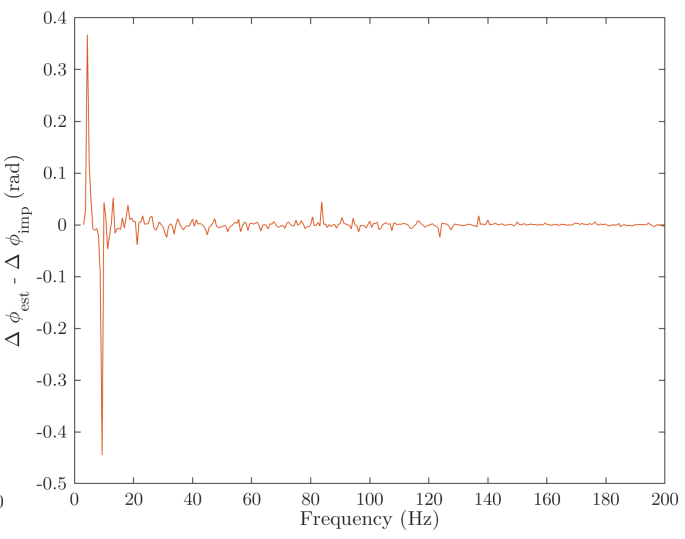

(b)

Figure 3: Phase differences for a single, $1.6 \mathrm{~s}$ data length, using the established [11], $\Delta \phi_{\text {est }}$, and improved, $\Delta \phi_{\text {imp }}$, frequency domain-based methods; a) phase differences and b) comparison between differences.

would be no need to develop the LDV capability for this purpose.

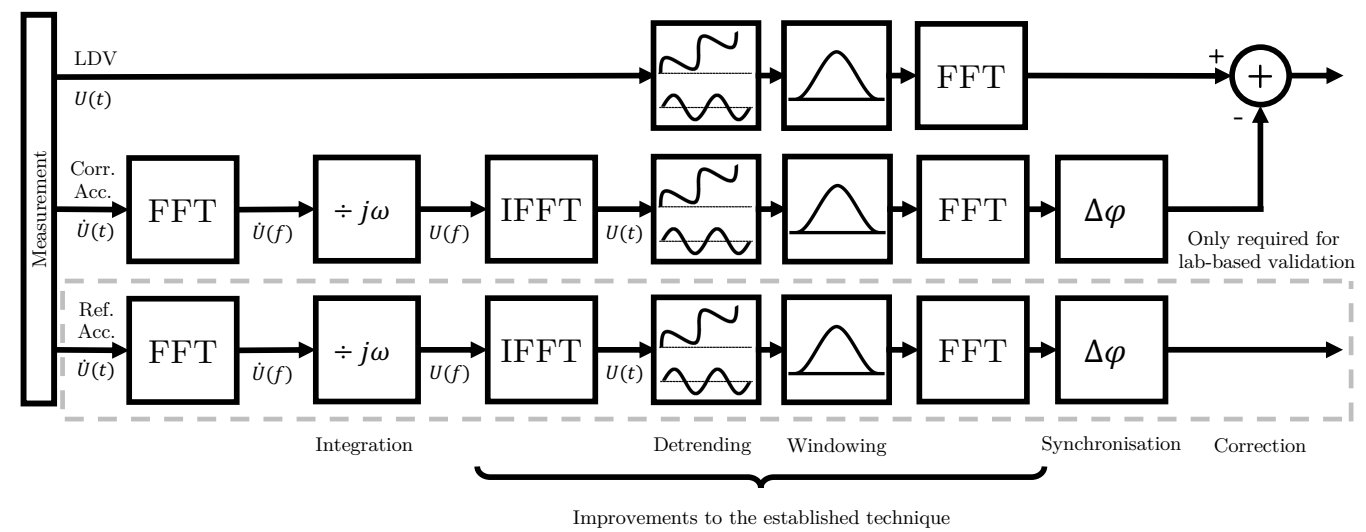

Figure 4: A functional diagram representing the improved frequency domain-based technique. The improvements are highlighted by the curly brackets and include the addition of the IFFT, detrending and FFT stages, along with moving the Windowing stage from after the measurement block to just before the second FFT. The signal "Corr. Acc." and "Ref. Acc." represent that of the correction and reference accelerometers, respectively. be subject to the same detrending step. Otherwise, the corrected LDV signal 
contains some signal content that has been removed from the detrended accelerometer signals. Following the second FFT, the correction processing is similar to that previously described [11], the exception being that, here, the accelerometer signals are already in velocity. Incorporating the previously determined time delays, before subtracting the correction accelerometer signal from the LDV (in complex representation), yields the corrected LDV signal for direct comparison with the 'true' vibration, given by the reference channel. The correction performance can be quantified using the error reduction, given by [15]:

$$
R=-10 \log _{10}\left(\frac{\mathrm{MSE}_{\mathrm{corr}}}{\mathrm{MSE}_{\text {meas }}}\right) \mathrm{dB}
$$

where $\mathrm{MSE}_{\text {meas }}$ and $\mathrm{MSE}_{\text {corr }}$ are the mean square error of the LDV signal before and after correction, respectively, when taking the processed reference accelerometer signal as the 'true' vibration signal.

Previously, $\mathrm{MSE}_{\text {meas }}$ and $\mathrm{MSE}_{\text {corr }}$ have been derived assuming there is no DC offset in the signal [15]. However, a complete description of the MSE for a signal of $N$ spectral lines and for the $m$ th spectra would be:

$$
\begin{aligned}
\mathrm{MSE}_{m}^{\text {signal }}= & \left(a_{0, m}^{\text {signal }}-a_{0, m}^{\text {true }}\right)^{2}+ \\
& \frac{1}{2} \sum_{n=1}^{N}\left(A_{n, m}^{\text {signal }}-A_{n, m}^{\text {true }}\right)^{2}+\left(B_{n, m}^{\text {signal }}-B_{n, m}^{\text {true }}\right)^{2}
\end{aligned}
$$

where $A_{n, m}^{\text {signal }}$ and $B_{n, m}^{\text {signal }}$ are the real and imaginary parts, respectively, of either the measured or corrected LDV signal at the $n$th spectral line for the $m$ th spectra The same notation applies to $A_{n, m}^{\text {true }}$ and $B_{n, m}^{\text {true }}$, which are the reference accelerometer equivalents. Similarly, $a_{0, m}^{\text {signal }}$ and $a_{0, m}^{\text {true }}$ are the DC component equivalents. When averaging across multiple spectra, the error reduction takes the following form:

$$
R=-10 \log _{10}(\bar{r}) \mathrm{dB}
$$

with:

$$
\bar{r}=\frac{1}{M} \sum_{m=1}^{M} \frac{\mathrm{MSE}_{m}^{\mathrm{corr}}}{\mathrm{MSE}_{m}^{\text {meas }}}
$$

where $M$ is the total number of acquired spectra. 


\subsection{Measurement correction performance comparison}

It is useful to visualise the performance difference between the two techniques as a function of the frequency. To do this, the error reduction can be calculated for each spectral line and plotted. The MSE in Eq. (2) can be substituted for the square error, SE, to preserve the frequency information. This plot is improved if a mean of each spectral line, $n$, is taken across the multiple spectra, $m$. Algebraically, this is given by:

$$
\begin{array}{rlr}
\mathrm{SE}_{\text {signal }}(n) & =\frac{1}{M} \sum_{m=1}^{M}\left(a_{0, m}^{\text {signal }}-a_{0, m}^{\text {true }}\right)^{2} & \text { for } n=0 \\
& =\frac{1}{2 M} \sum_{m=1}^{M}\left(A_{n, m}^{\text {signal }}-A_{n, m}^{\text {true }}\right)^{2}+\left(B_{n, m}^{\text {signal }}-B_{n, m}^{\text {true }}\right)^{2} & \text { for } n>0
\end{array}
$$

where all symbols are as previously defined. Substituting the SE in place of the MSE in Eq. (2) would then give:

$$
R(n)=-10 \log _{10}\left(\frac{\mathrm{SE}_{\text {corr }}}{\mathrm{SE}_{\text {meas }}}\right) \mathrm{dB}
$$




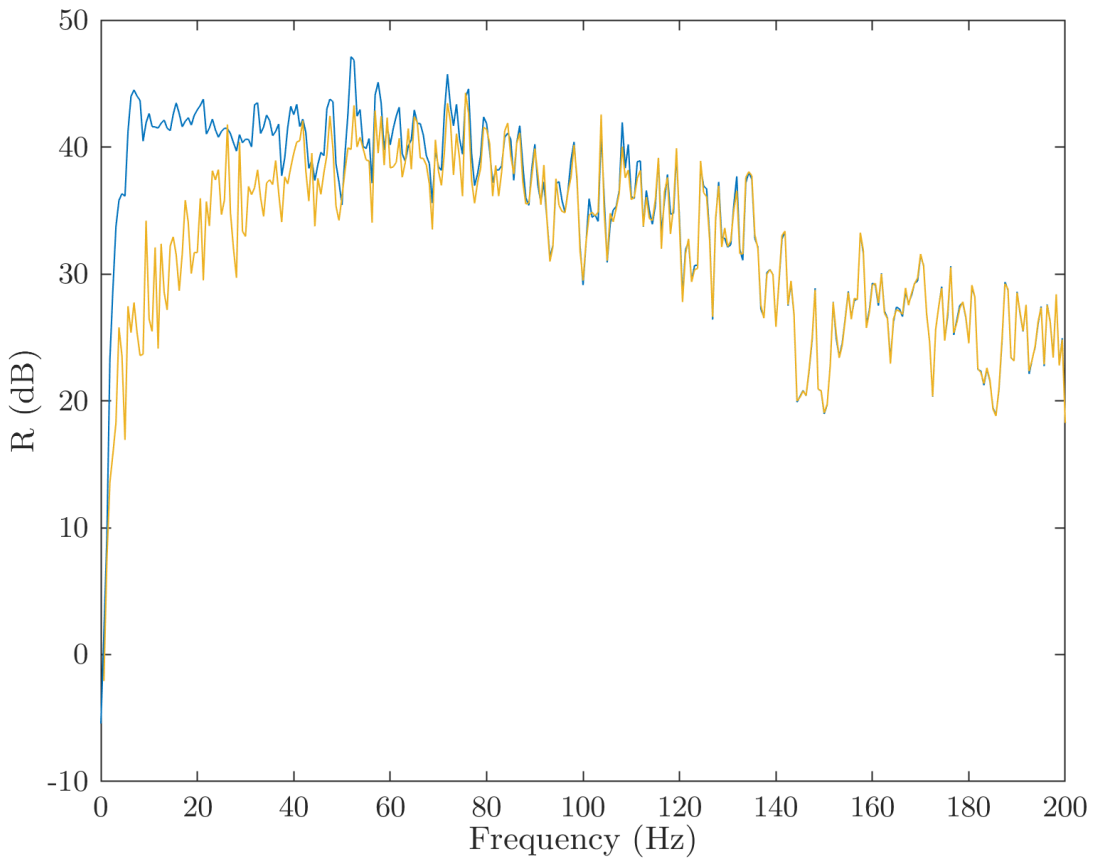

Figure 5: A plot of $R(f)$ obtained from $R(n)$ for both the established [11] and the improved frequency domain-based methods using five $1.6 \mathrm{~s}$ data lengths.

To obtain the data shown in Fig. 5, the reference channels for both techniques were processed identically and according to the technique presented in Fig. 4. These data show that the improved frequency domain-based technique outperforms the established technique for frequencies below $100 \mathrm{~Hz}$. However, for frequencies above $100 \mathrm{~Hz}$, the difference is less noticeable and this is expected since the effect of the detrending is focused at lower frequencies. Quantitatively, this improvement translates to a seven times increase in $R$, with the established and the improved techniques obtaining $25.0_{-1.3}^{+1.8} \mathrm{~dB}$ and $33.5_{-0.9}^{+1.2} \mathrm{~dB}$, respectively.

While the correction performance was already substantial, this further improvement clearly shows the value of detrending after signal integration and is important for several reasons. Firstly, it yields a technique with much improved lower frequency performance which is likely to be beneficial for a range of important applications where low frequency, low-level vibrations are likely. Secondly, it resolves an important discrepancy previously observed when comparing frequency domain processing with a novel time domain- 
based approach [16]. It should be noted that while previous work showed an eight times difference in the performance, the seven times difference in performance is not contradictory as the error reduction is dependant on the relative levels of the target and instrument vibration, therefore, is not consistent across setups. With performance inconsistency now resolved, the focus of the remainder of this paper will be a detailed assessment of the performance of the alternative time domain-based approach.

\section{Theoretical generalisation of the synchronisation error on the performance}

This section will work to establish an analytical model which relates the synchronisation error, $\Delta \tau$, to the quality of the correction. There will be a focus on time domain-based processing since the quality of the temporal alignment is limited to integer multiples of the time step. While interpolation could be used to upsample time domain data and enable sub-time step alignment, this is not always desirable. Therefore, this model can be used to predict the specifications of a system required to obtain high-quality time domain results based on the sampling frequency and the measured time delay.

\subsection{Relating the error reduction to the synchronisation error}

To relate the synchronisation error, $\Delta \tau$, to the error reduction, $R$, it is assumed that $\Delta \tau$ is the primary factor which affects the quality of the corrected velocity estimate, $\mathrm{MSE}_{\text {corr }}$. While other factors may also affect $\mathrm{MSE}_{\text {corr }}$, this model is not concerned with them. Therefore, a relationship between $\mathrm{MSE}_{\text {corr }}$ and $\Delta \tau$ is required to relate $\Delta \tau$ to $R$.

To do so, the corrected LDV signal, $v_{\text {corr }}(t)$, can be written as follows:

$$
v_{\text {corr }}(t)=v_{\text {meas }}(t)-v_{\text {acc }}(t)
$$

where $v_{\text {meas }}(t)$ is the target velocity measured by the LDV and $v_{\text {acc }}$ is the velocity of the LDV instrument itself, measured by the correction accelerometer. Rewriting Eq. (8a) to encapsulate the synchronisation error expressed as $v_{\text {corr }}^{\prime}(t)$ :

$$
v_{\text {corr }}^{\prime}(t)=v_{\text {meas }}(t)-v_{\text {acc }}(t+\Delta \tau)
$$


The velocity error, $\Delta v_{\text {corr }}(t)$, can then be defined as the difference between $v_{\text {corr }}(t)$ and $v_{\text {corr }}^{\prime}(t)$ :

$$
\Delta v_{\text {corr }}(t)=v_{\text {acc }}(t)-v_{\text {acc }}(t+\Delta \tau)
$$

Now a discrete Fourier expansion can be applied and, since $\Delta \tau$ is small, the small angle approximation can also be applied:

$$
\begin{gathered}
v_{a c c}(t)=\frac{a_{0}}{2}+\sum_{n=1}^{N} A_{n} \cos \left(n \omega_{0} t\right)+B_{n} \sin \left(n \omega_{0} t\right) \\
v_{\text {acc }}(t+\Delta \tau)=\frac{a_{0}}{2}+\sum_{n=1}^{N} A_{n}\left(\cos \left(n \omega_{0} t\right)-n \omega_{0} \Delta \tau \sin \left(n \omega_{0} t\right)\right) \\
+B_{n}\left(\sin \left(n \omega_{0} t\right)+n \omega_{0} \Delta \tau \cos \left(n \omega_{0} t\right)\right)
\end{gathered}
$$

where $A_{n}$ and $B_{n}$ are constants for each spectral line, $a_{0}$ is the DC component and $\omega_{0}$ is the spectral resolution. Substituting these expansions back into Eq. (9) and simplifying the expression gives:

$$
\Delta v_{\text {corr }}(t)=\Delta \tau \sum_{n=1}^{N}-A_{n} n \omega_{0} \sin \left(n \omega_{0} t\right)+B_{n} n \omega_{0} \cos \left(n \omega_{0} t\right)
$$

Therefore, $\mathrm{MSE}_{\mathrm{corr}}$, or $\overline{\Delta v_{\text {corr }}(t)^{2}}$, can be expressed as:

$$
\mathrm{MSE}_{\mathrm{corr}}=\frac{\Delta \tau^{2}}{2} \sum_{n=1}^{N}\left(A_{n} n \omega_{0}\right)^{2}+\left(B_{n} n \omega_{0}\right)^{2}
$$

Inevitable sources of error other than synchronisation error mean that the MSE will never be zero in practice. To account for this, an additional term, $c$, is introduced:

$$
\mathrm{MSE}_{\mathrm{corr}}=c+\frac{\Delta \tau^{2}}{2} \sum_{n=1}^{N}\left(A_{n} n \omega_{0}\right)^{2}+\left(B_{n} n \omega_{0}\right)^{2}
$$

where $c$ is a constant representing the lowest practically obtainable MSE with a given setup. In order to relate this to the mean error reduction, Eq. (2) can be rearranged into the following form: 


$$
\frac{\mathrm{MSE}_{\text {corr }}}{\mathrm{MSE}_{\text {meas }}}=10^{-\frac{R}{10}}=r
$$

where $r$ has been defined for convenience. Combining this with Eq. (13), the following can be written:

$$
r(\Delta \tau)=\frac{c+\frac{\Delta \tau^{2}}{2} \sum_{n=1}^{N}\left(A_{n} n \omega_{0}\right)^{2}+\left(B_{n} n \omega_{0}\right)^{2}}{\mathrm{MSE}_{\text {meas }}}
$$

The value of $r(\Delta \tau=0)$ can then be described as "optimal", and denoted by $r_{\text {opt }}$. Therefore, Eq. (15) can also be written as:

$$
r(\Delta \tau)=r_{\mathrm{opt}}+K \Delta \tau^{2}
$$

where $r_{\text {opt }}$ and $K$ have now incorporated all remaining constants. Both $r_{\text {opt }}$ and $K$ can be experimentally determined for a given setup.

Finally, an expression for $R(\Delta \tau)$ can be written by substituting Eq. (16) into Eq. (2):

$$
R(\Delta \tau)=-10 \log _{10}\left(r_{\text {opt }}+K \Delta \tau^{2}\right)
$$

However, to make use of this relationship, the synchronisation error must be derived and this differs for the frequency and time domain.

\subsection{Frequency domain synchronisation error}

The synchronisation error in the frequency domain, $\Delta \tau_{f}$, is simple since it only depends on how accurately the signal delay is known. Mathematically this can be defined as:

$$
\Delta \tau_{f}=\tau_{\text {meas }}-\tau_{\text {true }}
$$

where $\tau_{\text {meas }}$ is the measured time delay and $\tau_{\text {true }}$ is the theoretical true time delay. Practically, $\tau_{\text {true }}$ is the theoretical unknowable exact true time delay between the signals and $\tau_{\text {meas }}$ is determined using the procedure outlined in Section 3.1. Substituting this into Eq. (17) gives the following:

$$
R\left(\tau_{\text {meas }}\right)=-10 \log _{10}\left(r_{\text {opt }}+K\left(\tau_{\text {meas }}-\tau_{\text {true }}\right)^{2}\right)
$$

Therefore, this frequency domain model predicts there will be no sampling frequency dependence of the performance. 


\subsection{Time domain synchronisation error}

The synchronisation error in the time domain, $\Delta \tau_{t}(d t)$, is not only dependant on the accuracy of the measured time delay, but also on the time step. This is given by:

$$
\Delta \tau_{t}(d t)=\left\lfloor\frac{\tau_{\text {meas }}}{d t}\right\rceil d t-\tau_{\text {true }}
$$

370

where $d t$ is the time step and $\lfloor\ldots\rceil$ denotes the nearest integer. Eq. (20a) can now be written in terms of the sampling frequency, $f_{s}$, instead of the time step since that is the adjustable acquisition parameter:

$$
\Delta \tau_{t}\left(f_{s}\right)=\left\lfloor\tau_{\text {meas }} f_{s}\right\rceil \frac{1}{f_{s}}-\tau_{\text {true }}
$$

Since $\tau_{\text {true }}$ is the theoretically true value, it bares little practical significance. Moving forward, it will be assumed that $\tau_{\text {meas }} \approx \tau_{\text {true }}$ so that the effect of the sampling frequency alone on the error reduction can be thoroughly assessed; both will now be denoted as $\tau$. This assumption also results in both positive and negative synchronisation errors having an equivalent negative effect on the velocity estimate. Combining Eq. (17) and Eq. (20b) gives:

$$
R\left(f_{s}\right)=-10 \log _{10}\left(r_{\mathrm{opt}}+K\left(\left\lfloor\tau f_{s}\right\rceil \frac{1}{f_{s}}-\tau\right)^{2}\right)
$$

The general form of the time domain model, with the significant features labelled, can be seen in Fig. 6. As can be seen therein, there are three distinct regions. The first, "Inactive Region", displays no sensitivity to the sampling frequency. In this region the time step, $d t$, is too small for any temporal alignment to take place; as such, no performance change occurs. Temporal alignment becomes possible once the time step is smaller than $\frac{1}{2 \tau}$, representing the beginning of the "Transitionary Region". This region is characterised by a large increase in the performance, as the decreasing time step allows for increasingly more accurate temporal alignment. The third and final region, the "Oscillatory Region", is characterised by oscillations in performance which decrease in amplitude as the frequency increases. The peaks of these occur at integer multiples of $\tau^{-1} \mathrm{~Hz}$, since these locations are where $\tau$ becomes divisible by an integer number of time steps, leading to an 
increase in the accuracy of the temporal alignment. Similarly, the performance troughs occur at integer multiples of $0.5 \tau^{-1} \mathrm{~Hz}$

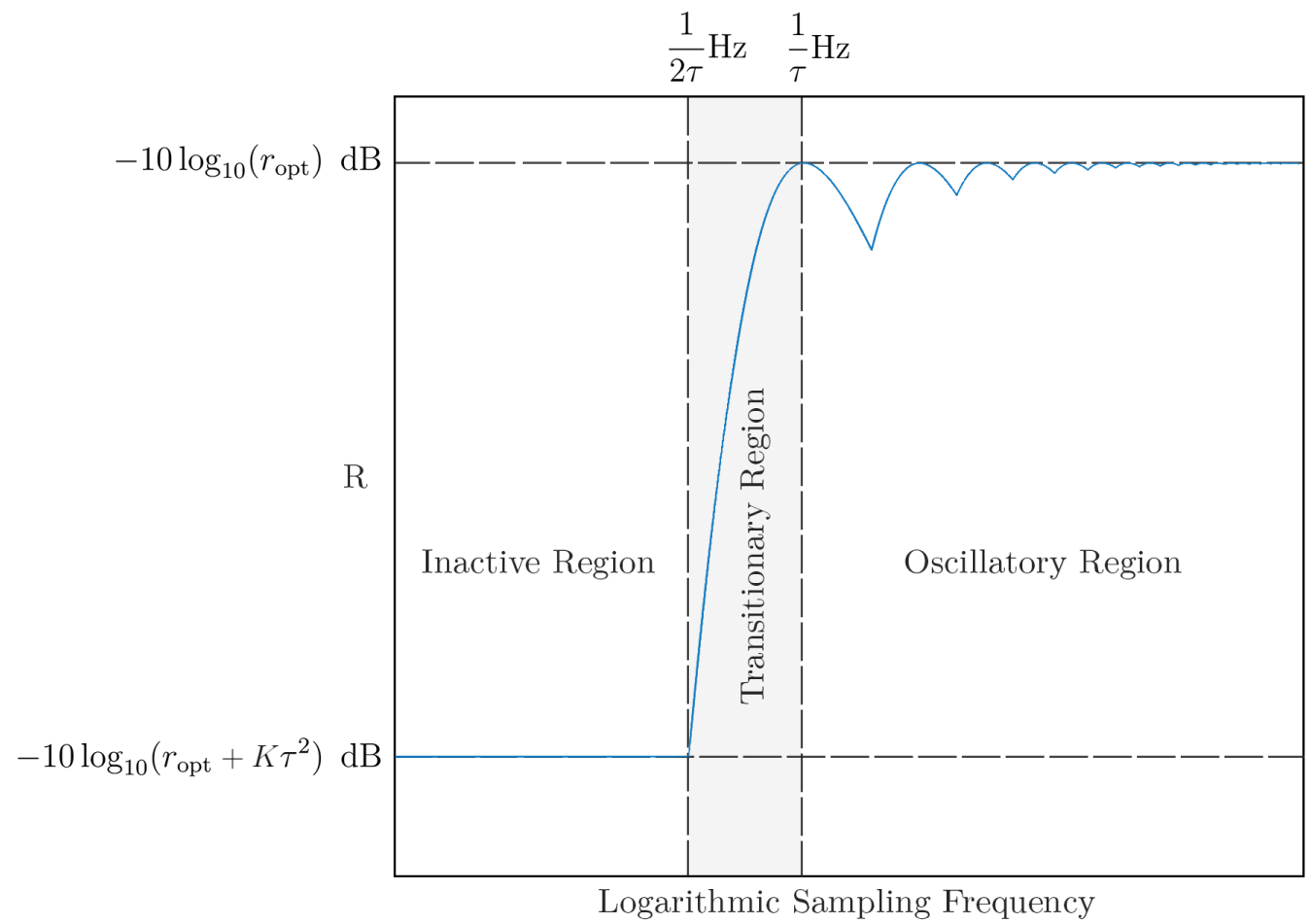

Figure 6: A general plot of the time domain error reduction model as a function of the sampling frequency with three distinct regions labelled. Higher values on the vertical scale represent better performance.

Since a continuous range of sampling frequencies is rarely available, a more practically relevant example is Eq. (21) plotted at the sampling frequencies available on the Simcenter SCADAS Mobile data acquisition system, as seen in Fig. 7. While the highest sampling frequency, $204.8 \mathrm{kHz}$, shows a significant reduction in error, the same performance could have been achieved by using $8.192 \mathrm{kHz}, 16.384 \mathrm{kHz}$ or $40.960 \mathrm{kHz}$. In fact, the aforementioned frequencies have a error reduction $0.03 \mathrm{~dB}$ higher than the highest sampling frequency. This shows that, even without measuring values for the constants $K$ and $r_{\text {opt }}$, the time domain model can still predict the optimal sampling 
404

frequency to maximise performance.

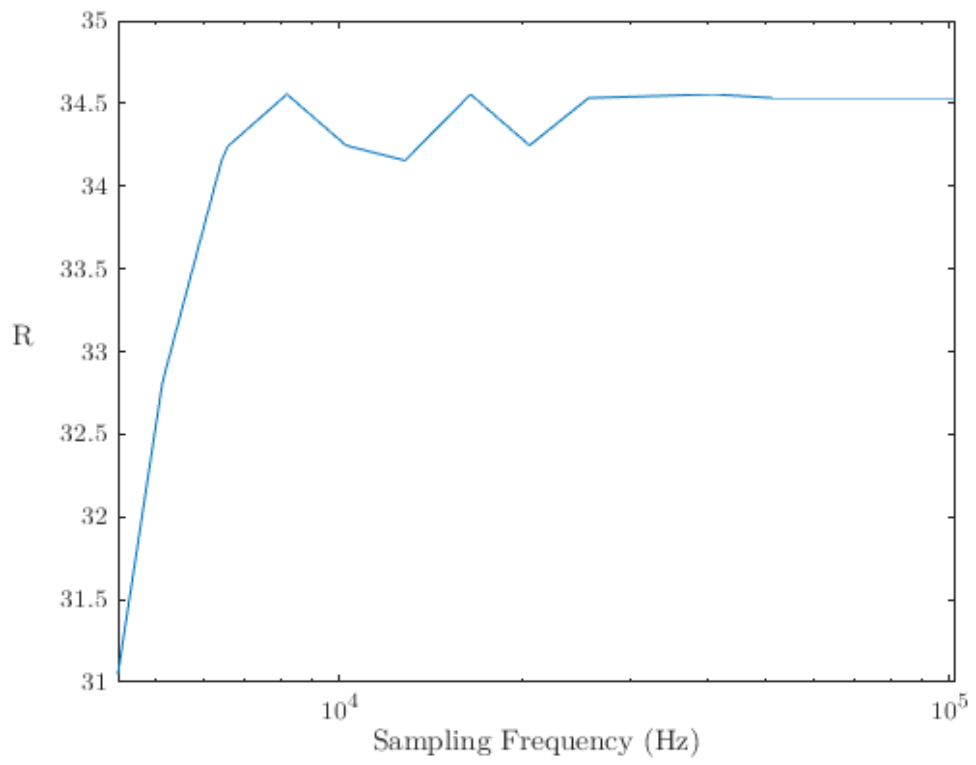

Figure 7: A plot of error reduction as a function of the sampling frequencies available on the Simcenter SCADAS Mobile data acquisition system. This is plotted with values of $K=35 \times 10^{3} \mathrm{~s}^{-2}$ and $r_{\mathrm{opt}}=350 \times 10^{-6}$.

\subsection{Time domain constants determination}

Since the main use of the time domain model is to enable the informed selection of the sampling frequency, knowledge of the constants is not necessary. However, in order to validate the time domain model it will be compared to an experimentally measured $R\left(f_{s}\right)$, denoted by $R_{\mathrm{ex}}\left(f_{s}\right)$, meaning the constants are required since they affect the model's relative proportions in the vertical axis.

The first constant, $r_{\mathrm{opt}}$, is calculated using:

$$
r_{\text {opt }}=10^{-\frac{\max \left(R_{\text {ex }}\right)}{10}}
$$

where $\max \left(R_{\text {ex }}\right)$ is the maximum value of $R_{e x}(f)$. Similarly, $K$, can be calculated using: 


$$
K=\frac{r_{\mathrm{opt}}-10^{-\frac{R_{\mathrm{ex}}\left(f_{s}\right)}{10}}}{\left(\left\lfloor\tau_{\text {meas }} f_{s}\right] \frac{1}{f_{s}}-\tau_{\text {meas }}\right)^{2}} \quad \text { for } \quad f_{s}>\frac{1}{2 \tau_{\text {meas }}} \mathrm{Hz}
$$

where all symbols are as previously defined. The sampling frequency here must be larger than $\frac{1}{2 \tau_{\text {meas }}} \mathrm{Hz}$ as the time domain model does not predict any behaviour in the Inactive Region so scaling using these data will lead to erroneous predictions.

\section{Experimental validation of the time domain model}

This section has two aims: firstly, to show that the improvements made to the established frequency domain-based technique close the performance gap when compared to the time domain-based technique; secondly, to show their relative performances over a range of frequencies and, by doing so, confirm the time domain model for $R\left(f_{s}\right)$, given by Eq. (21). This will provide the user with two instrument vibration correction techniques when faced with stationary and transient signal types. The experimental arrangement used in the following was common with that used in Section 2.

\subsection{Data collection and processing}

To validate the time domain model presented in Eq. (23), the error reduction needs to be characterised against the sampling frequency and thus data is acquired at the highest available sampling frequency of $204.8 \mathrm{kHz}$ and iteratively downsampled to simulate acquisition at lower sampling frequencies. The process was implemented in MATLAB and Fig. 8 illustrates this code for the time domain and improved frequency domain-based techniques. 
Iterate with $D=1,2,3, \ldots, 400$
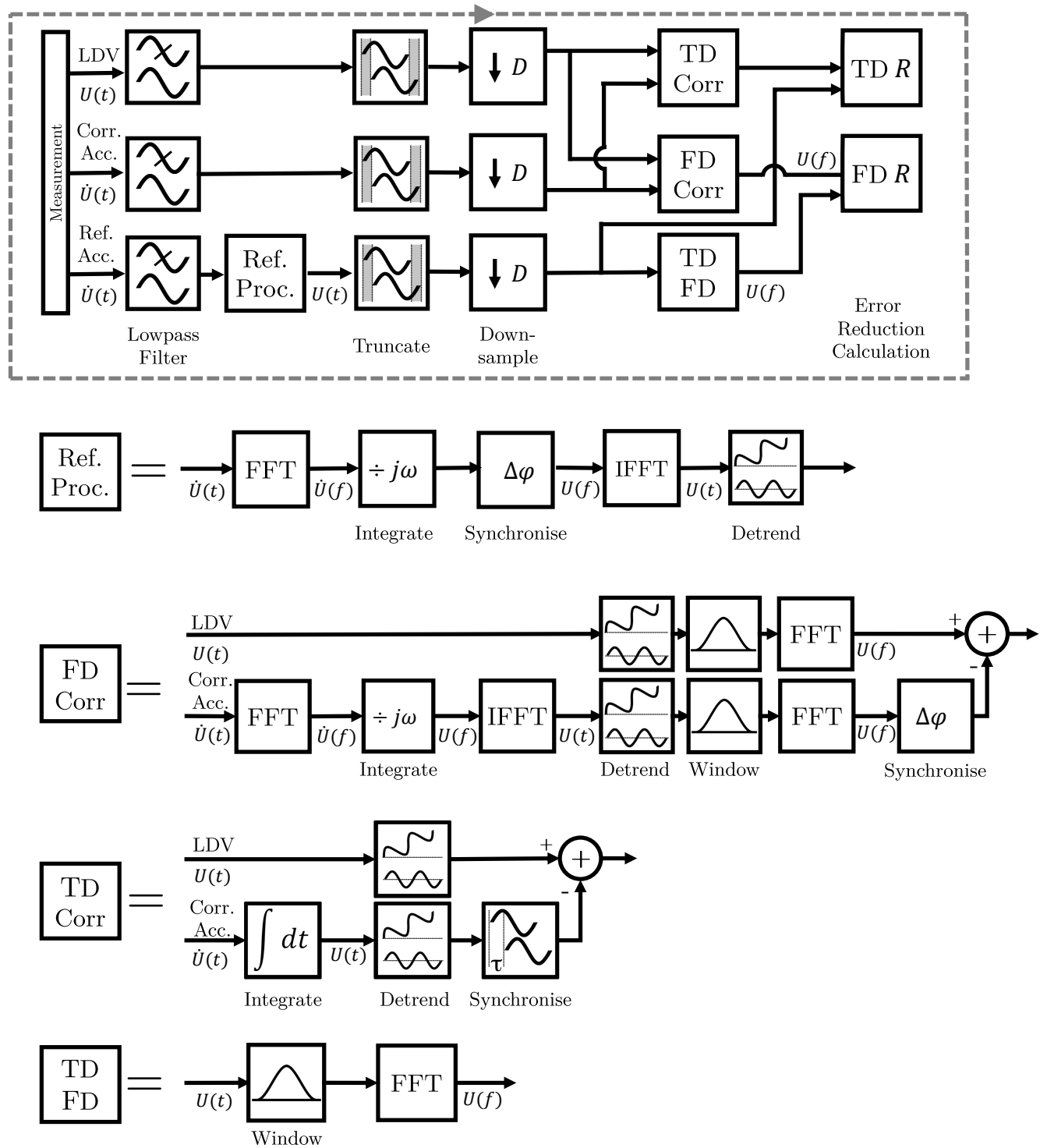

Figure 8: A schematic of the code used to characterise the error reduction as a function of sampling frequency. Where $D$ is a downsampling factor and "TD Corr" and "FD Corr" represent both the time domain [16] and improved frequency domain-based correction techniques, respectively.

As shown in Fig. 8, measured data are immediately low-pass filtered using a finite impulse response digital lowpass filter with a $200 \mathrm{~Hz}$ cut-off frequency which coincides with the maximum frequency of the vibration. Any spuri- 
ous higher frequency signal content which might otherwise have been aliased into the frequency range of interest following the downsampling is thereby rejected. Following this, the reference accelerometer signal is then subjected to the same frequency domain detrending and time synchronisation steps as that previously described. This special treatment is present purely to ensure that the reference signal is as close to a 'true' signal as possible, in terms of both the integration accuracy and synchronisation error.

Since the Fourier Transform implicitly assumes that a signal is periodic, the phase shift would have caused a portion of the signal at the beginning or the end of the reference accelerometer signal to wrap around to the opposite end of the signal, depending on which signal is lagging. To fix this, all samples in this 'wrapped' region are removed from all three transducer signals in the stage named "Truncation" shown in Fig. 8. Following this, the signals are downsampled by taking each $D$ th sample from the original signals, simulating a lower sampling frequency acquisition. The penultimate stages named "TD Corr" and "FD Corr" represent the two correction algorithms. This process was looped in the code with $D=1,2, \ldots, 400$, giving a minimum sampling frequency of $512 \mathrm{~Hz}$ and a total of 400 data points for each correction technique. The final output is, therefore, two data sets describing the performance of each correction algorithm as a function of the sampling frequency.

\subsection{Model validation and sample rate dependent performance assessment}

The frequency domain model, given by Eq. (19), predicts no sampling frequency dependence as sub-time step synchronisation is possible in the frequency domain. However, the time domain model, given by Eq (21), predicts a reasonably strong dependence due to this time step synchronisation limitation. Comparing Figures 9 and 6, the three previously defined regions are clearly identifiable from the experimental data. In particular, the important Transitionary Region is clearly shown; this is where the performance of the time domain-based technique "overtakes" that of the frequency domainbased approach. 


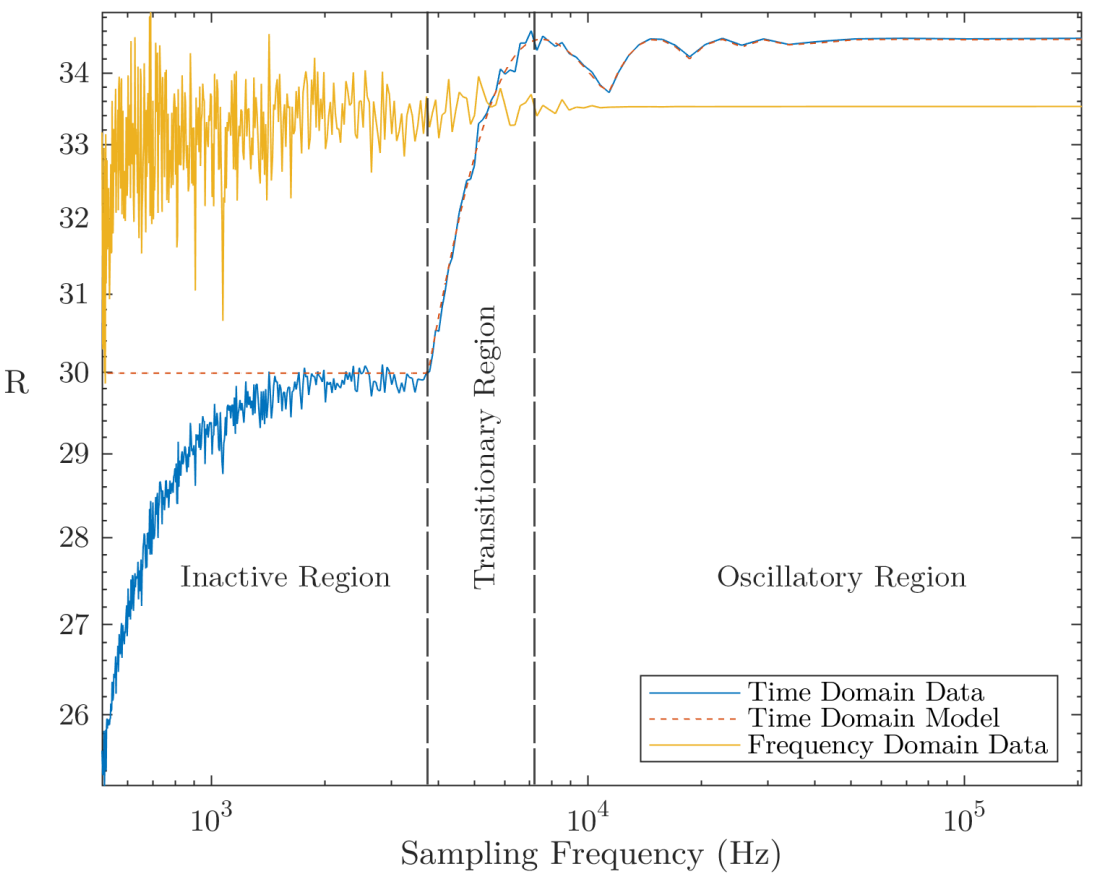

Figure 9: A plot of the experimental error reduction as a function of the sampling frequency for the improved frequency domain and the time domain-based techniques. The error reduction is calculated using Eq. (2) and the domain-specific formulations for the MSE. The time domain model, given by Eq. (21), is also plotted for validation purposes.

In order to validate the time domain model, the time domain data can be compared to the time domain model in Fig. 9; from this, two shortcomings can be seen. Firstly, the time domain model does not predict the smaller noise-like fluctuations visible at frequencies below $10 \mathrm{kHz}$ in the time domain data. However, these fluctuations are exhibited by both correction techniques and are likely caused by different factors as they do not occur at common frequencies. There was no attempt to model these fluctuations, therefore, this is no major shortcoming of either model. Secondly, the time domain model fails to predict the behaviour in the Inactive Region. The three times increase seen in the time domain experimental data is likely caused by a decrease in the quality of integration at the lower sampling frequencies when using the cumulative trapezoidal method. The time domain model does not capture this behaviour as it only considered the effects of temporal alignment.

However, as can be seen in Fig. 9, the time domain model almost ex- 
actly describes the experimental data in the Transitionary and Oscillatory Regions, which are the regions of interest here. With the time domain model validated, its value is its ability to determine when the time domain technique will perform optimally, based on the time delay estimate and the sampling frequency. That is, significant improvements will be made to the quality of the correction if a data acquisition system is used with a sampling frequency larger than $\frac{1}{2 \tau} \mathrm{Hz}$. Similarly, any sampling frequency larger than $\tau^{-1} \mathrm{~Hz}$ will not yield any substantial increase in performance and is therefore unnecessary. Also, if possible, a sampling frequency should be selected close to a performance peak located at $\frac{n}{2 \tau} \mathrm{Hz}$ with $n=1,2,3, \ldots$.

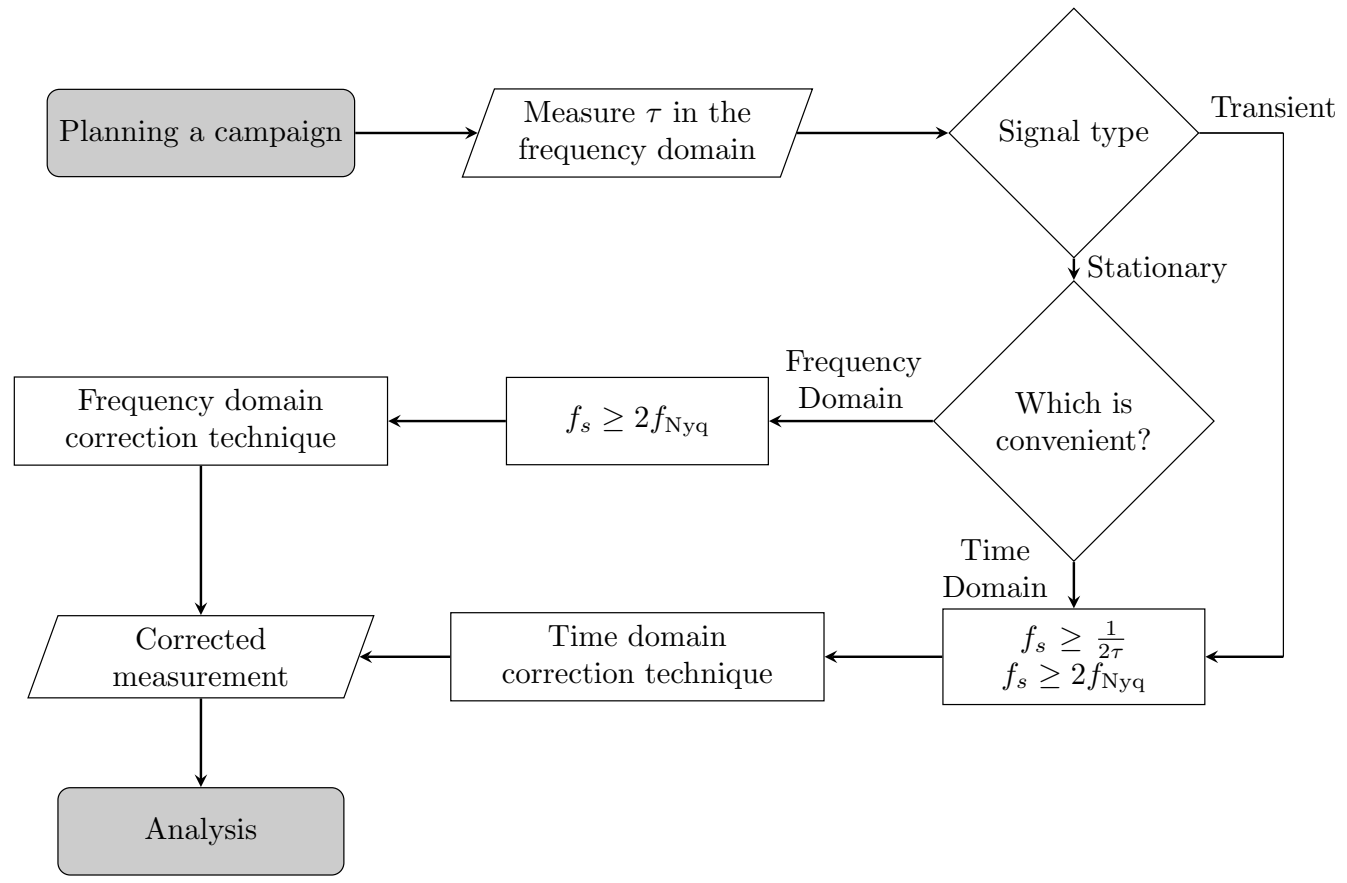

Figure 10: A flow chart describing when to use either the time domain [16] or the improved frequency domain-based technique described herein. The outcome is based on the sampling frequencies available to the user and $\tau$.

Comparing the performances of the two techniques in the region $f_{s}>\frac{1}{2 \tau}$ $\mathrm{Hz}$, as shown in Fig. 9, there is about a decibel of performance gain to be made by using the time domain-based technique. The exact reason for the difference in performance is yet to be determined. However, since this is 
small, either technique could be used with minimal difference in achieved performance. Similarly, the user could select a sampling frequency close to a performance peak to optimise the time domain-based technique performance. However, the benefit of this is also marginal. Fig. 10 summarises these generalised findings based on the vibration signal type and the sampling frequency, advising the user which is the most appropriate technique to use for a given measurement campaign. When the vibration is stationary in nature, the user can use either technique. When the vibration is transient in nature, the user must use the time domain-based technique and select an appropriate sampling frequency to optimise performance.

\section{Conclusions}

Recent advances in the application of LDVs to measurement campaigns in which the instrument sensor head is itself subject to vibration have lead to an increasing number of techniques for the correction of the measured signals. Practical implementation of these techniques involves the determination of the sensor head vibration and subtraction of this in post-processing. Extension from lab to field-based measurements has further necessitated the conception and development of novel time domain-based processing techniques for vibration signals that are transient in nature. Initial investigations showed that, for common signals, these alternative techniques significantly outperformed previously established frequency domain equivalents.

Firstly, therefore, this work aimed to close this previously observed performance gap with an improved frequency domain based technique being developed. A seven times performance increase was obtained by applying a modified frequency domain-based technique which included a detrending step prior to implementation of the correction processing. To make this detrending possible, it is necessary that no window is applied to the sampled data until after detrending. Particular improvement was shown to be found in frequencies below $100 \mathrm{~Hz}$ which is arguably a major benefit since applications of interest for such techniques are expected to be focused in this frequency range.

Since the required correction measurements are typically obtained using accelerometers, in addition to the requisite integration, it is typical that signal synchronisation is necessary due to signal conditioning differences. It is well known that the quality of the signal synchronisation will contribute to 
the correction performance. Therefore, when working in the time domain and when interpolation is not desirable, the sampling frequency would then contribute to the synchronisation error as time shifts are only possible in units of the time step. As such, a model describing the relationship between the synchronisation error and the performance was derived and formulated in terms of the sampling frequency and the error reduction. To validate this model, code was written to obtain the sampling frequency dependence of the performance by iteratively downsampling high-sample rate data to simulate acquired data at a range of sampling frequencies. The various correction techniques were then tested on these data to experimentally obtain the relationship derived in the model with excellent agreement found.

Given two viable and equally effective correction techniques, each with their own set of requirements, a framework was developed to allow the user to conveniently select the appropriate correction technique, taking into account the specifics of the vibration measurement of interest. Whichever the required technique, the significant contribution of this work is to enable the user to optimally specify the data acquisition parameters. This enables definition of the optimal hardware characteristics required for a given measurement campaign, important for efficient and practical integration of such sensor solutions.

\section{Acknowledgements}

The first author wishes to acknowledge the International Research Scholarship support from UTS. The authors wish to acknowledge the support of Robotic Systems Pty Ltd.

\section{References}

[1] S. J. Rothberg, M. S. Allen, P. Castellini, D. Di Maio, J. J. Dirckx, D. J. Ewins, B. J. Halkon, P. Muyshondt, N. Paone, T. Ryan, H. Steger, E. P. Tomasini, S. Vanlanduit, J. F. Vignola, An international review of laser Doppler vibrometry: Making light work of vibration measurement, Opt. Lasers Eng. 99 (2017) 11-22. https://doi.org/ 10.1016/j.optlaseng. 2016.10.023.

[2] L. A. Jiang, M. A. Albota, R. W. Haupt, J. G. Chen, R. M. Marino, Laser vibrometry from a moving ground vehicle, Appl. Opt. 50 (2011) 2263. https://doi.org/10.1364/A0.50.002263. 
[3] T.Writer, J. M. Sabatier, M. A. Miller, K. D. Sherbondy, Mine detection with a forward moving portable laser Doppler vibrometer, Proc. SPIE 4742 (2002) 649-653. https://doi.org/10.1117/12.479136.

[4] B. Libbey, D. Fenneman, B. Burns, Mobile platform for acoustic mine detection applications, Proc. SPIE 5794 (2005) 683. https: //doi.org/10.1117/12.603410.

[5] V. Aranchuk, A. Lal, J. M. Sabatier, Multi-beam laser Doppler vibrometer for landmine detection, Opt. Eng. 45 (2006) 104302. https: //doi.org/10.1117/1.2358975.

[6] A. Drabenstedt, X. Cao, U. Polom, F. Patzold, T. Zeller, P. Hecker, V. Seyfried, C. Rembe, Mobile seismic exploration, AIP Conf. Proc. 1740 (2016) 030001. https://doi.org/10.1063/1.4952659.

[7] S. W. Courville, P. C. Sava, Speckle noise attenuation in orbital laser vibrometer seismology, Acta Astronaut. 172 (2020) 16-32. https:// doi.org/10.1016/j.actaastro.2020.03.016.

[8] S. W. Courville, P. C. Sava, Speckle noise in orbital laser doppler vibrometry, Lunar Planet. Sci. Conf. 39 (2019) 697-699.

[9] P. Sava, E. Asphaug, Seismology on small planetary bodies by orbital laser Doppler vibrometry, Adv. Space Res. 64 (2019) 527-544. http: //doi.org/10.1016/j.asr.2019.04.017.

[10] M. A. A. Ismail, A. Bierig, S. R. Hassan, R. Kumme, P. T. Bundesanstalt, Flyable Mirrors : Laser Scanning Vibrometry Method for Monitoring Large Engineering Structures Using Drones, Proc. SPIE 11142 (2019) 5-8. http://doi.org/10.1117/12.2535570

[11] B. J. Halkon, S. J. Rothberg, Towards laser Doppler vibrometry from unmanned aerial vehicles, J. Phys. Conf. Ser. 1149 (2018) 012022. http://doi .org/10.1088/1742-6596/1149/1/012022.

[12] M. R. Moreu, Fernando., Taha, Railroad Bridge Inspections for Maintenance and Replacement Prioritization Using Unmanned Aerial Vehicles with Laser Scanning Capabilities, IDEA Prog. 18 (2018) 06761. 
[13] B. J. Halkon, S. J. Rothberg, Taking laser Doppler vibrometry off the tripod: correction of measurements affected by instrument vibration, Opt. Lasers Eng. 91 (2017) 16-23. http://doi.org/10.1016/j. optlaseng.2016.11.006

[14] B. J. Halkon, S. J. Rothberg, Restoring high accuracy to laser Doppler vibrometry measurements affected by vibration of beam steering optics, J. Sound Vib. 405 (2017) 144-157. https://doi.org/10.1016/j.jsv . 2017.05 .014 .

[15] B. J. Halkon, S. J. Rothberg, Establishing correction solutions for scanning laser Doppler vibrometer measurements affected by sensor head vibration, Mech. Syst. Signal Process. 150 (2021) 107255. https : //doi.org/10.1016/j.ymssp.2020.107255.

[16] A. Darwish, B. Halkon, S. Oberst, R. Fitch, S. Rothberg, Correction of laser doppler vibrometer measurements affected by sensor head vibration using time domain techniques, Proc. Int. Conf. Struct. Dyn., Eurodyn 2 (2020) 4842-4850. https://doi.org/10.47964/1120.9392. 20444.

[17] A. Brandt, R. Brincker, Integrating time signals in frequency domain - Comparison with time domain integration, Measurement 58 (2014) 511-519. https://doi.org/10.1016/j.measurement.2014.09.004.

[18] Endevco, Variable capacitance accelerometer datasheet - Model 770A770F, https://buy.endevco.com/ContentStore/MktgContent/ Endevco/Datasheets/EDV-DS-770A-770F_lowres.pdf, 2020 (accessed 30th July 2021).

[19] P. Martin, S. J. Rothberg, Pseudo-vibration sensitivities for commercial laser vibrometers, Mech. Syst. Signal Process. 25 (2011) 2753-2765. https://doi.org/10.1016/j.ymssp. 2011.02.009. 
Click here to access/download LaTeX Source Files elsarticle-harv.bst

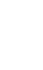




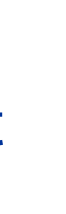

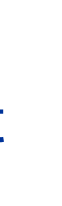

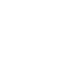


Click here to access/download LaTeX Source Files elsarticle-num.bst 
Click here to access/download

\section{LaTeX Source Files}

\section{model1-num-names.bst}


Click here to access/download

\section{LaTeX Source Files} JSV.tex 


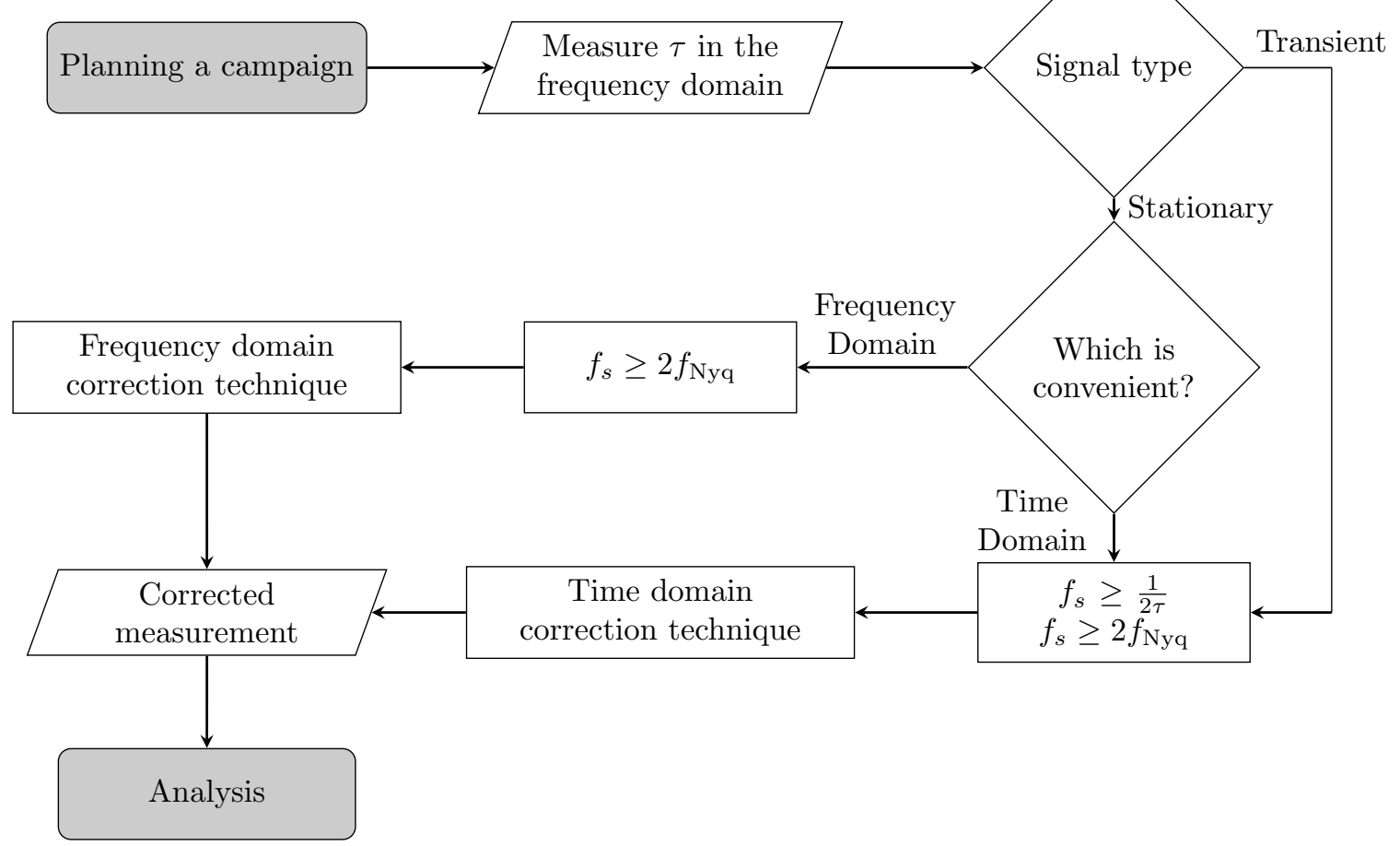




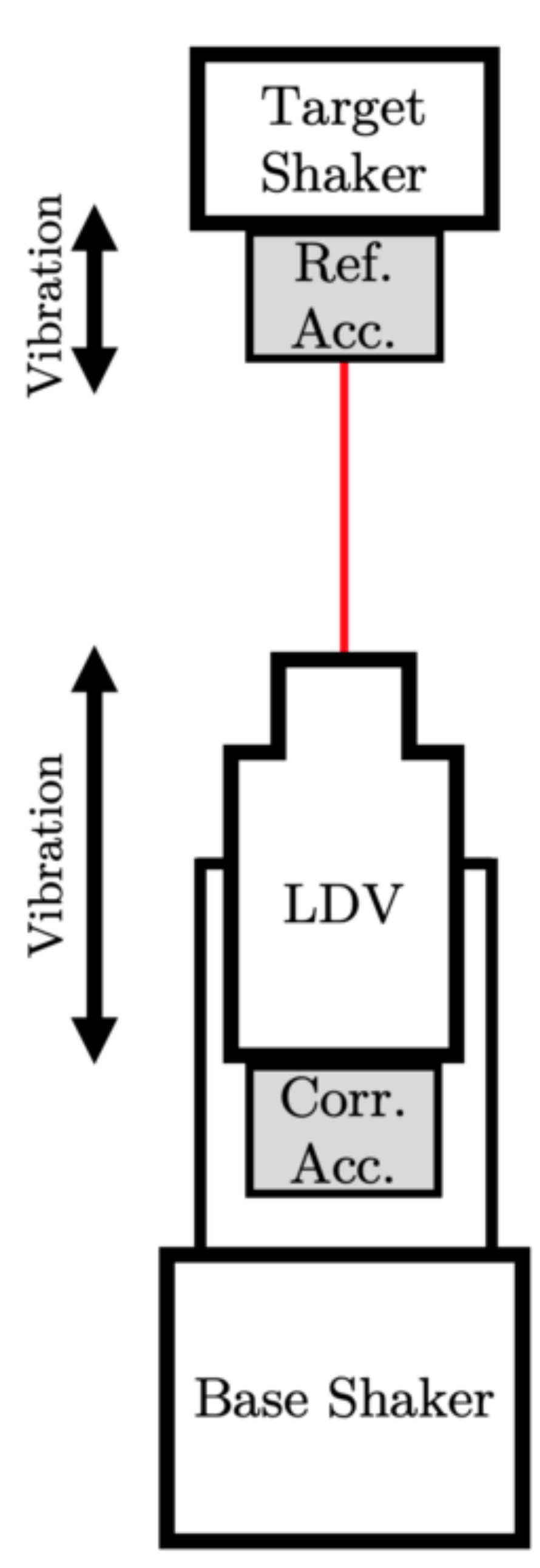

Fig 1a

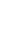

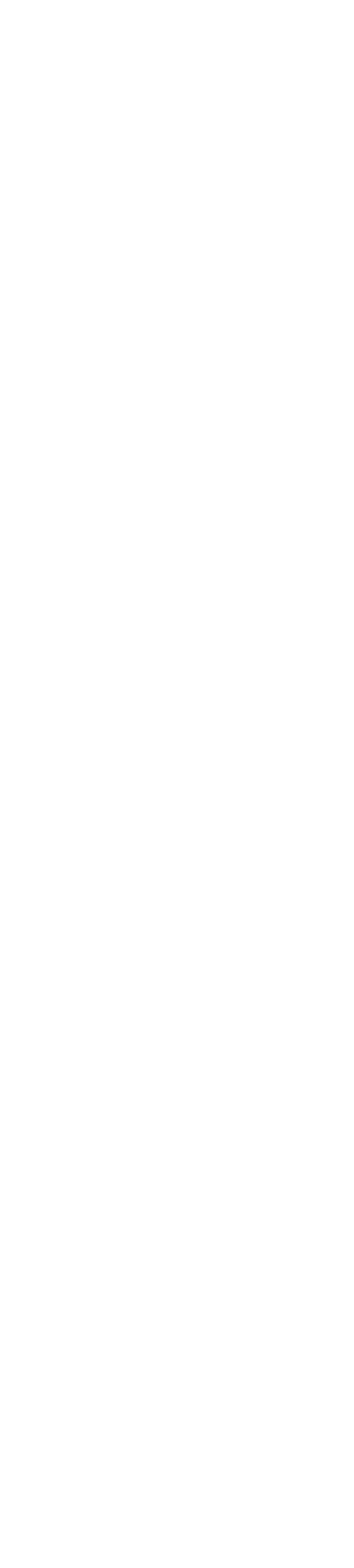

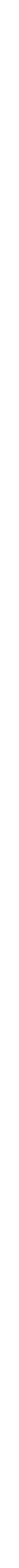




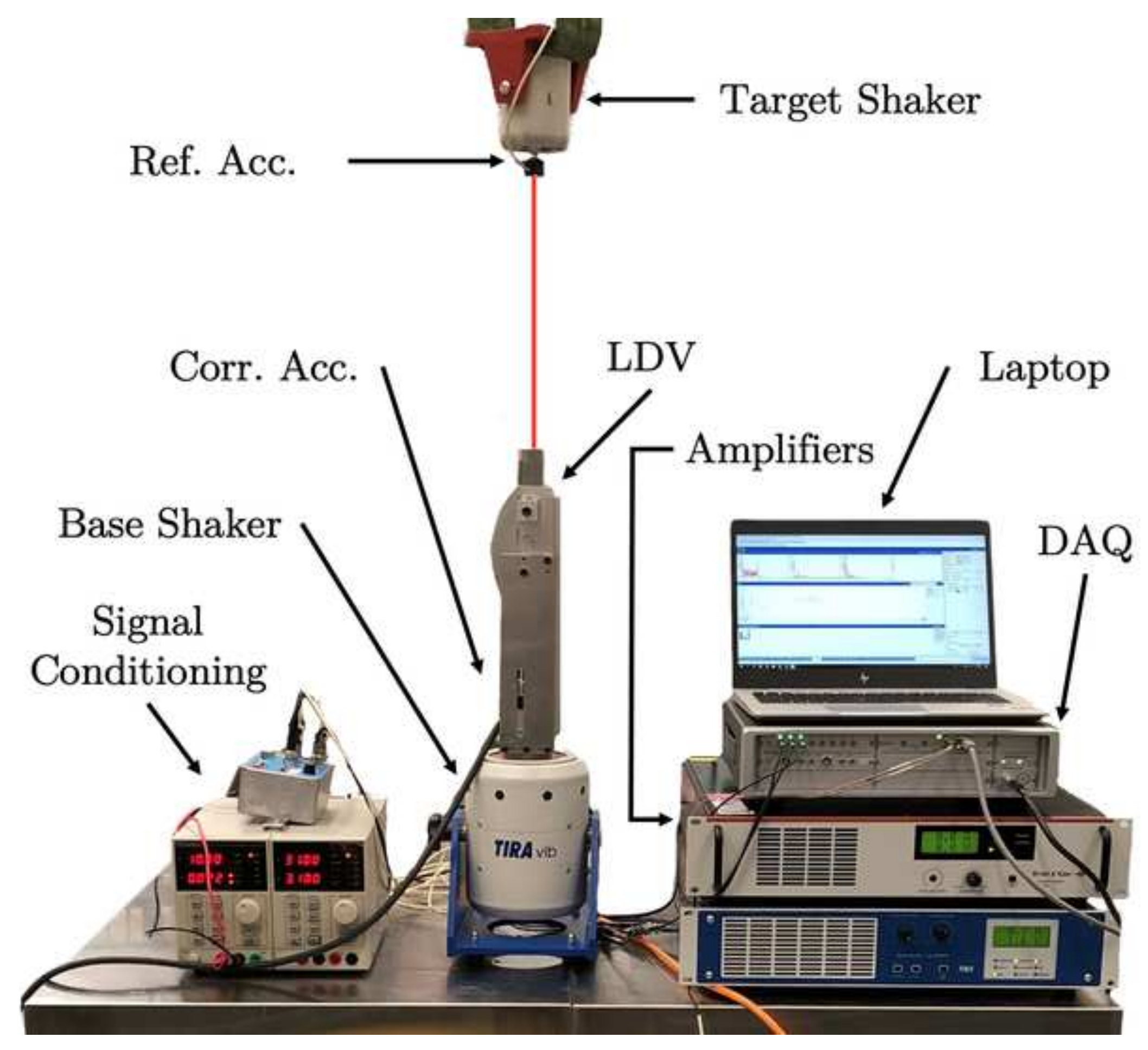




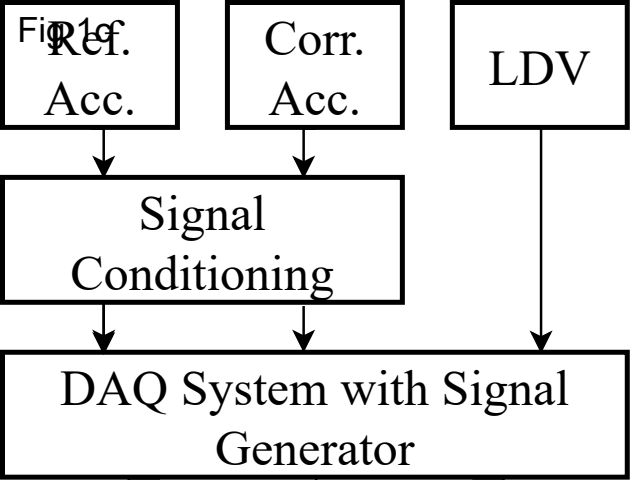


Click here to access/download;Figure(s);fig3a.png $\underline{\underline{ \pm}}$

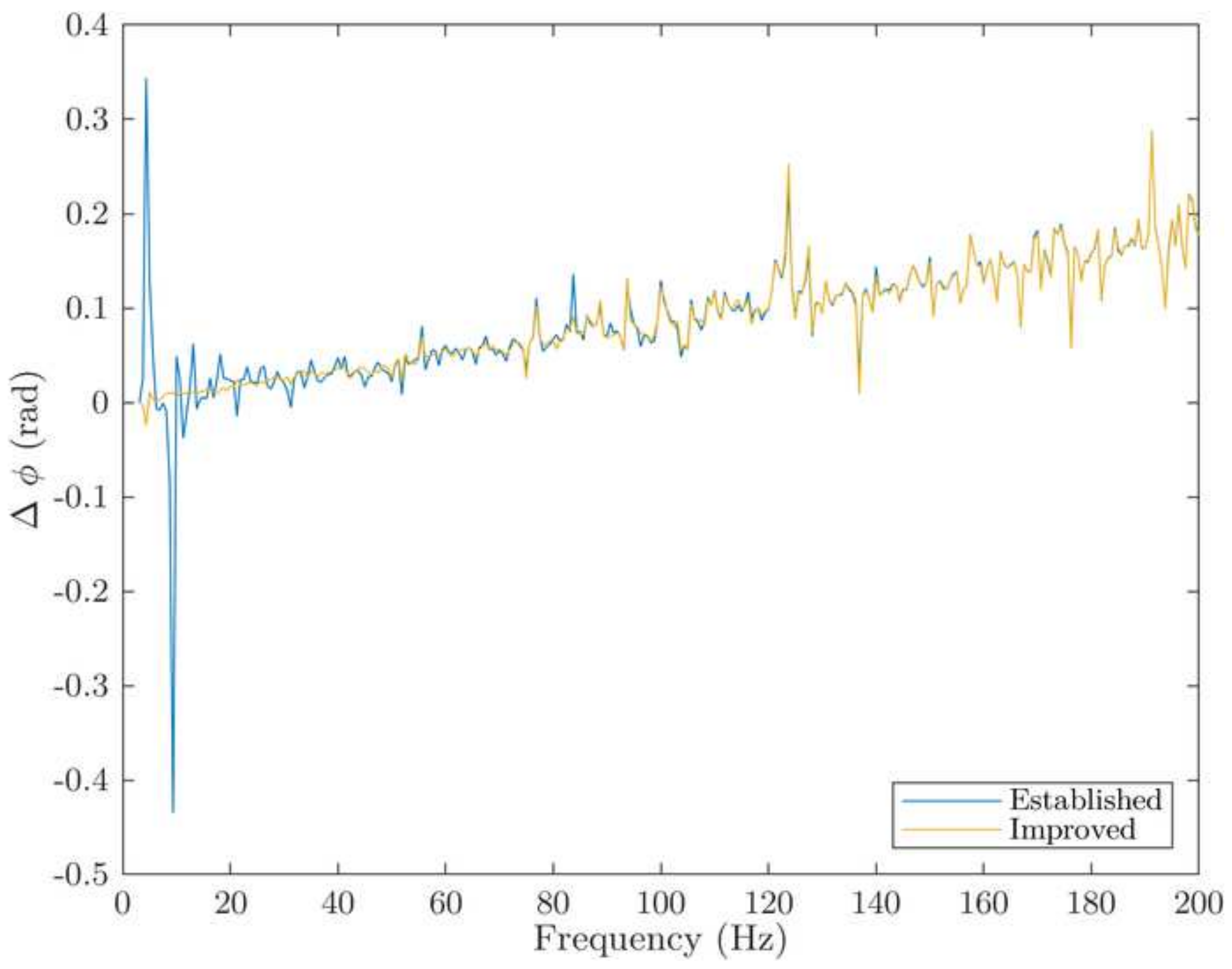


Click here to access/download;Figure(s);fig3b.png $\underline{\underline{ \pm}}$

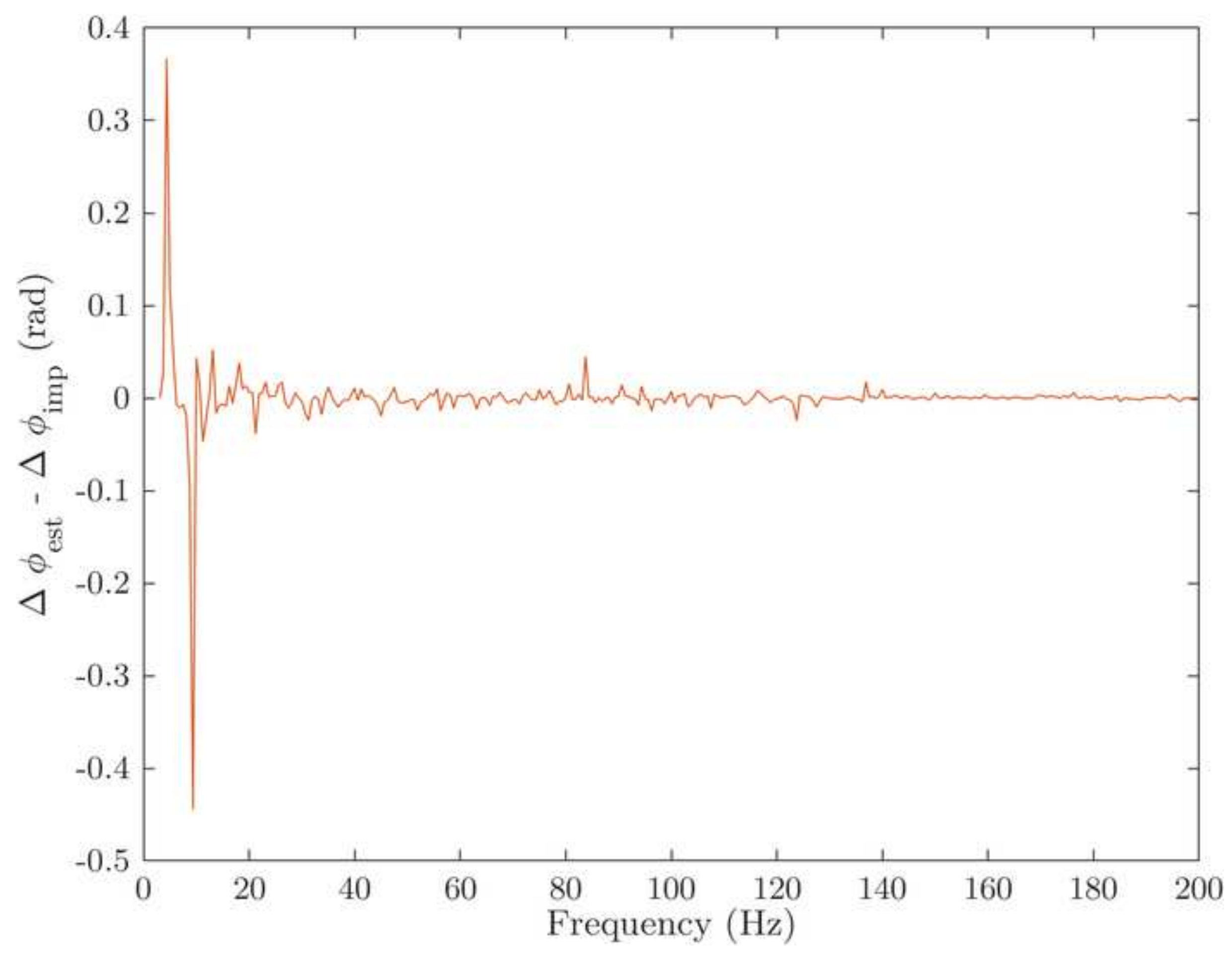


Click here to access/download;Figure(s);fig5.png $\underline{\underline{ \pm}}$

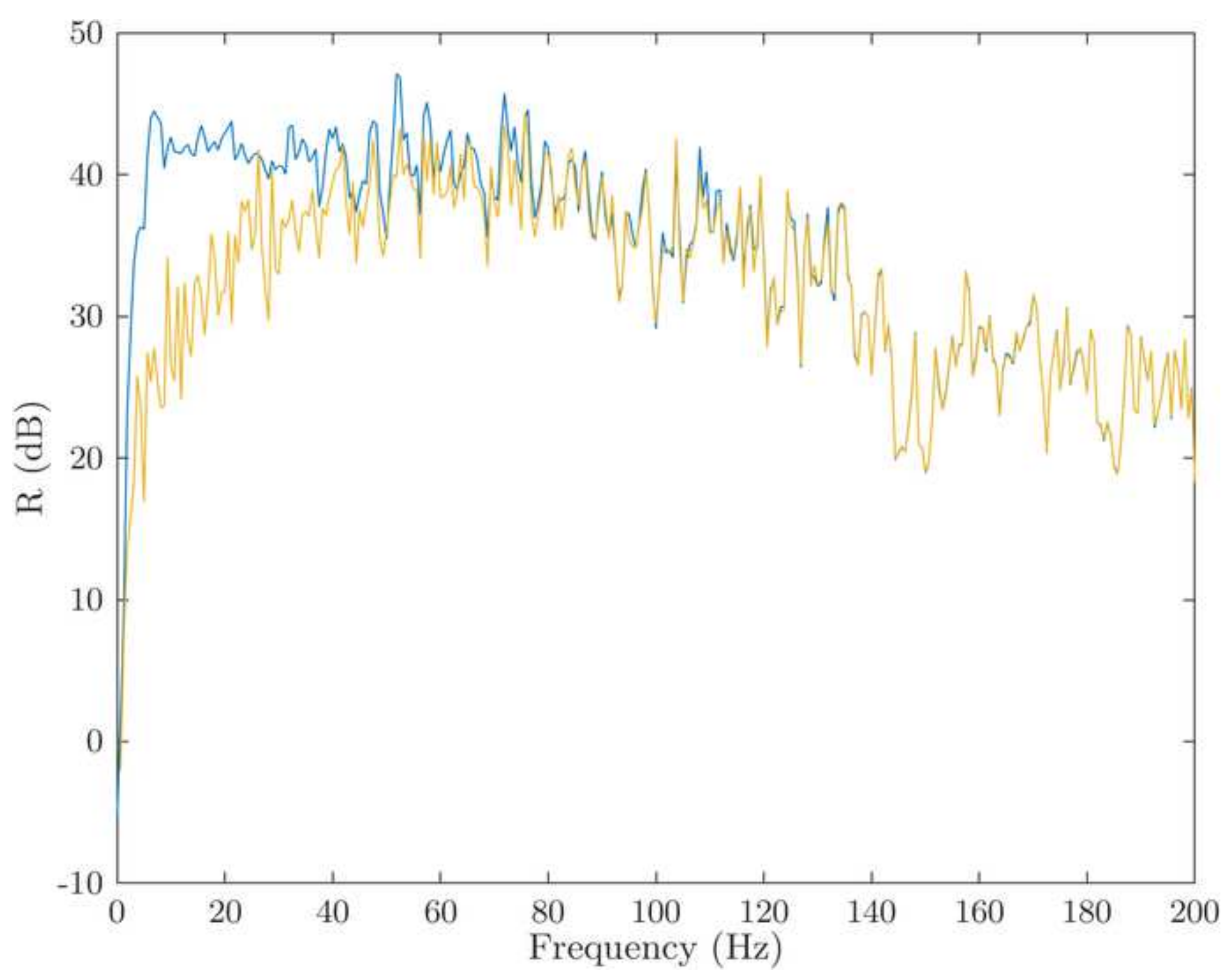




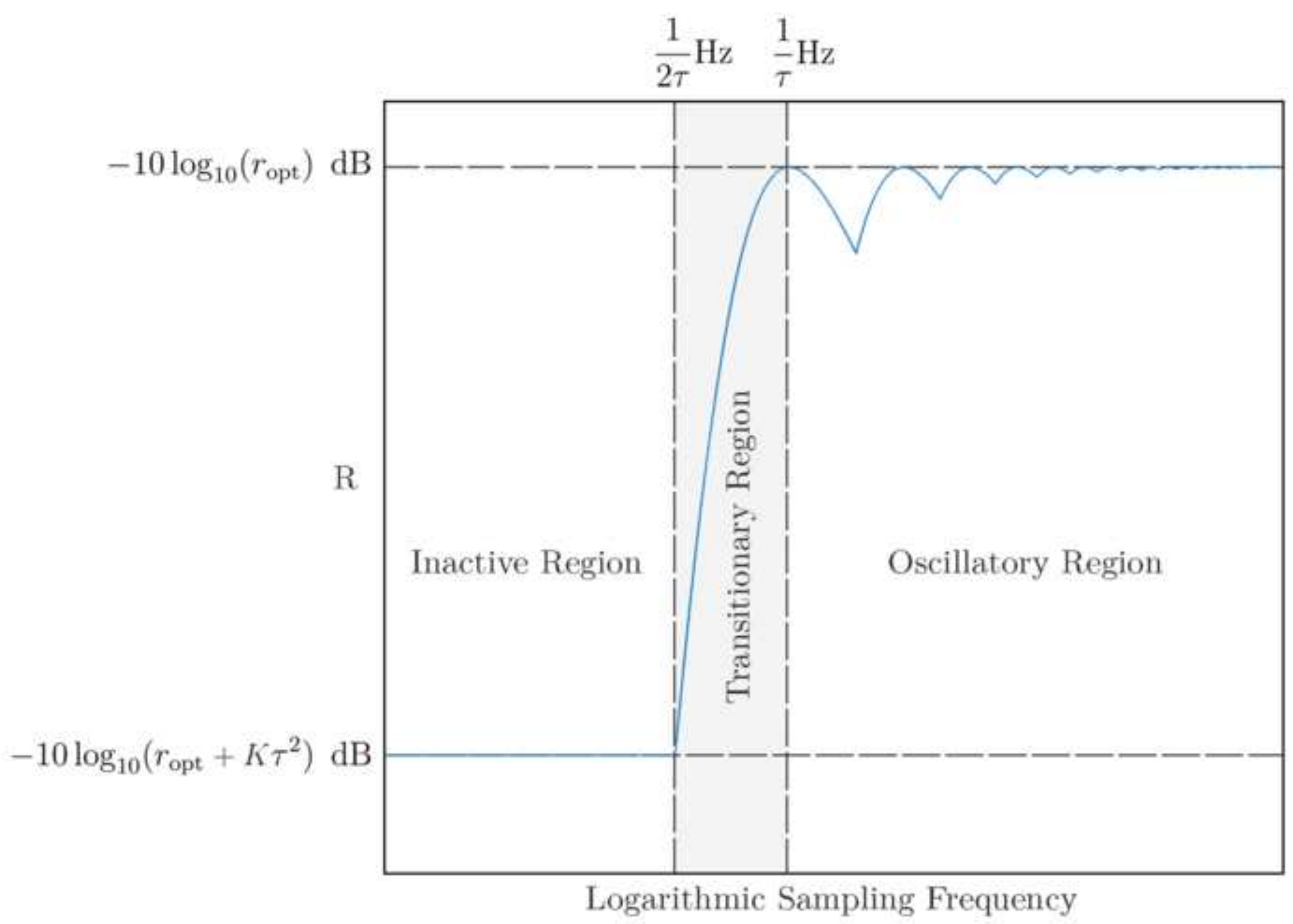


Click here to access/download;Figure(s);fig7.png $\underline{\underline{ \pm}}$

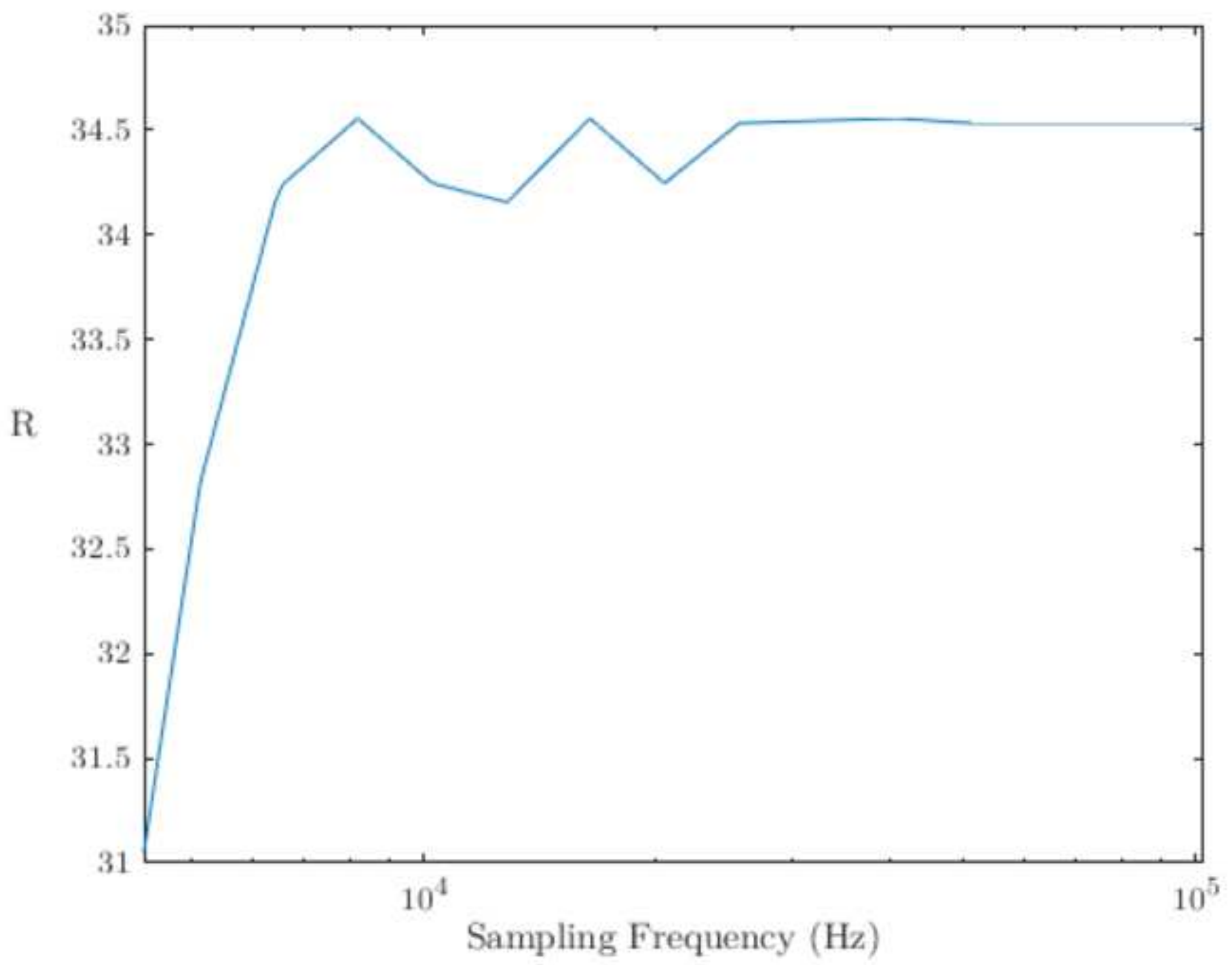


Iterate with $D=1,2,3, \ldots, 400$
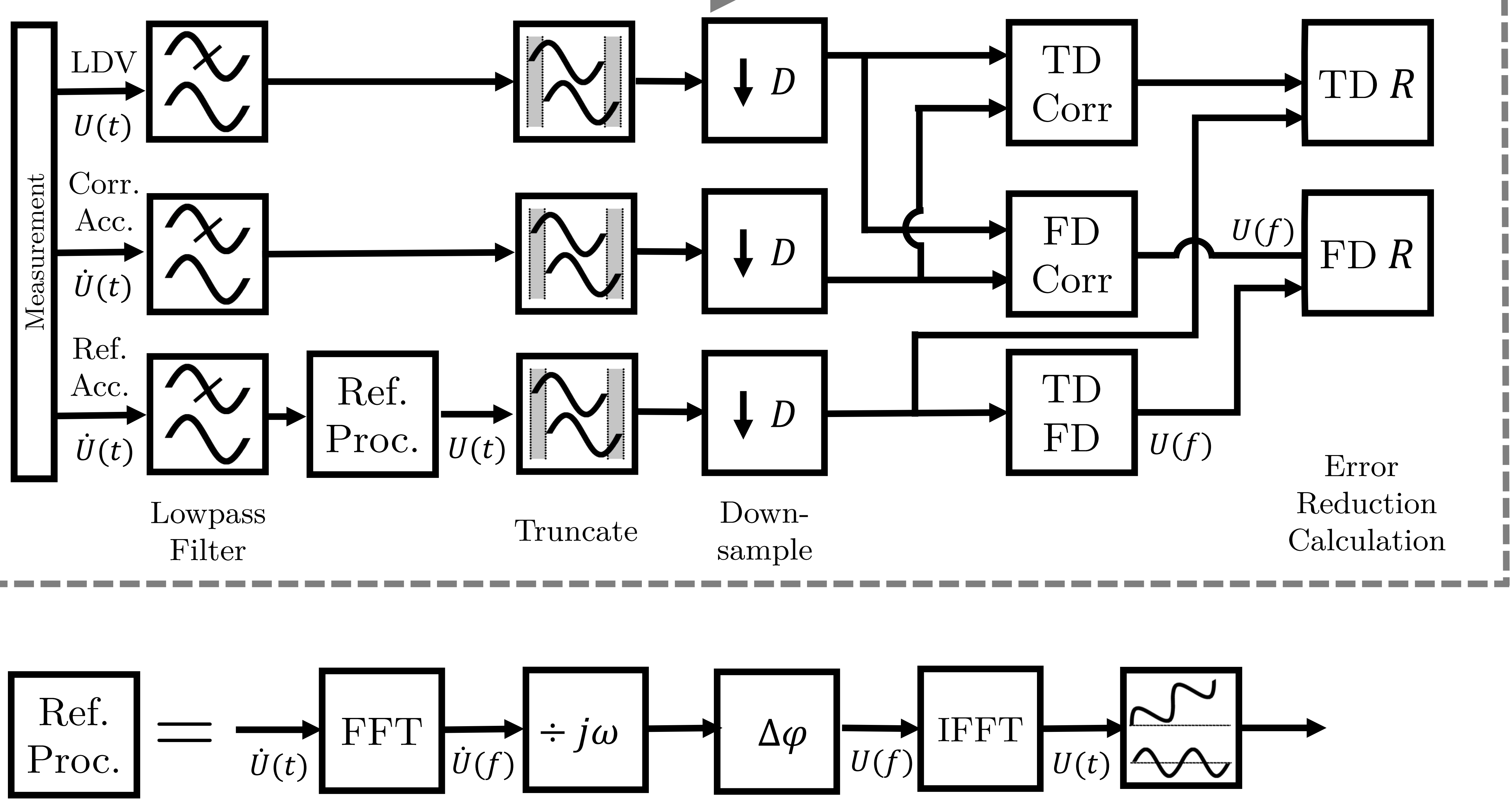

Integrate Synchronise

Detrend

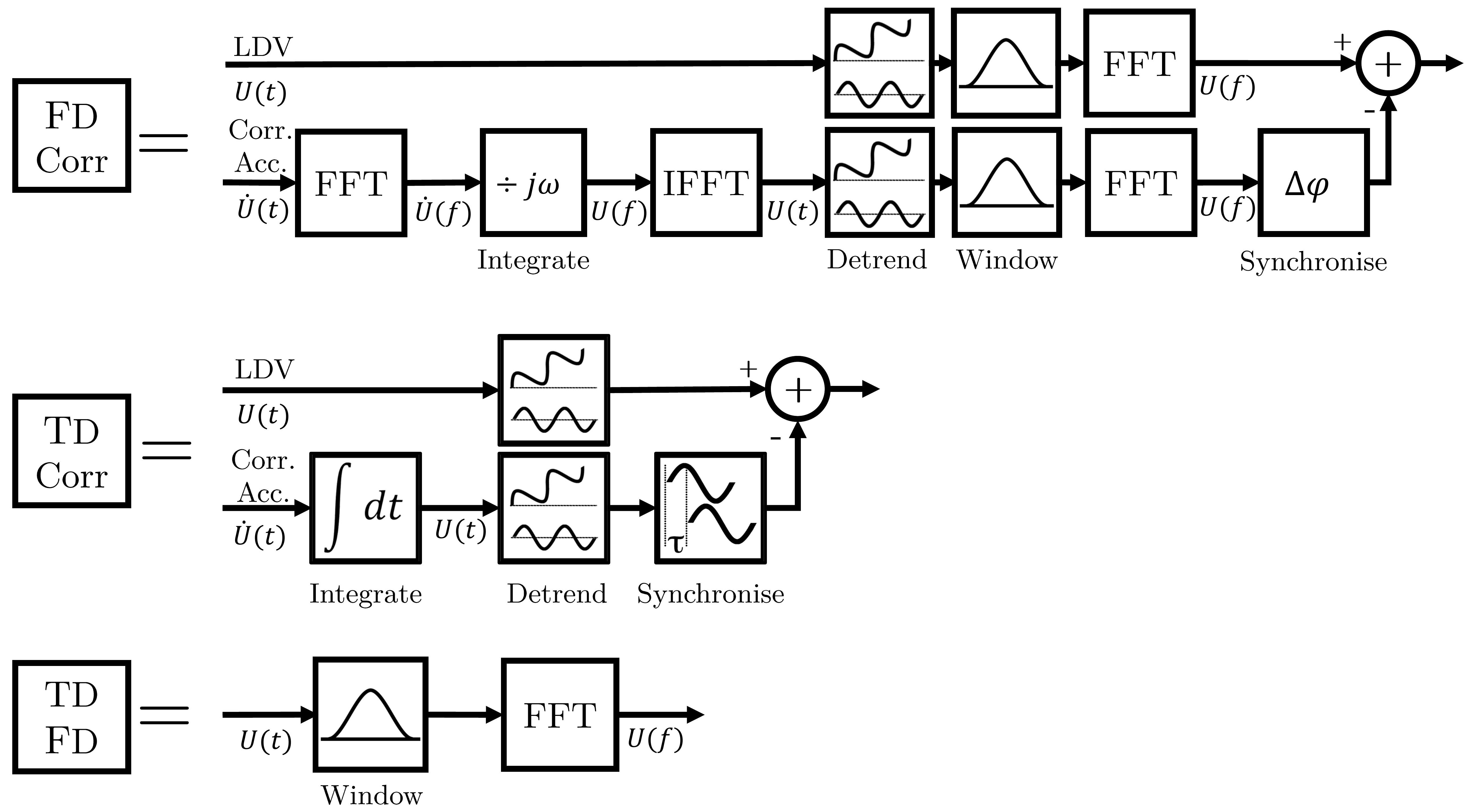


Click here to access/download;Figure(s);fig9.png $\underline{\underline{ \pm}}$

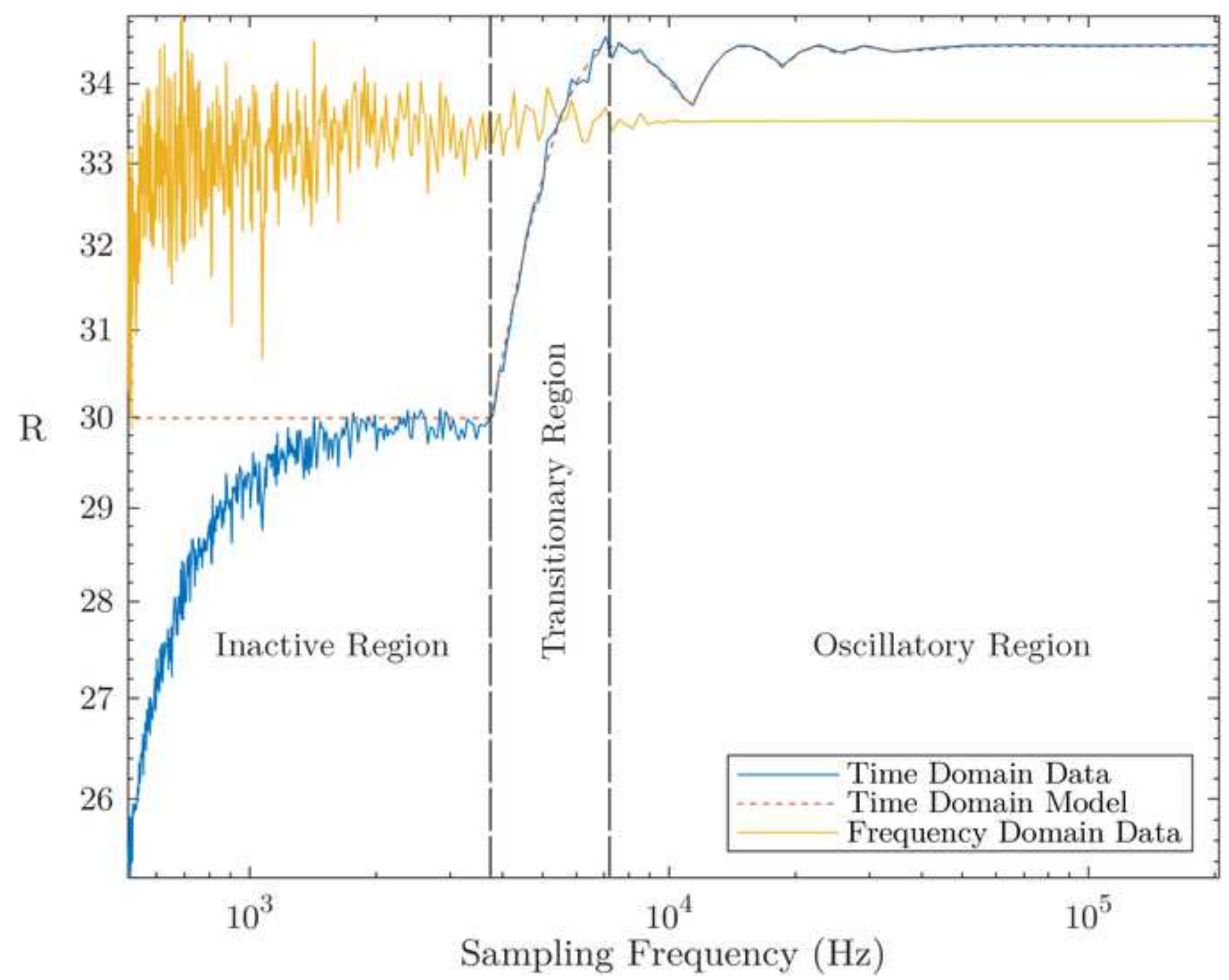


Journal of Sound and Vibration

Author Checklist

Authors should complete the following checklist and submit with their revised manuscript.

Math notation follows requirements on Guide for Authors (GFA) see:

https://www.elsevier.com/journals/journal-of-sound-and-vibration/0022-460X/guide-for-authors

Use Roman (normal upright) type for: Total differential operators (e.g. $d$ in differential); i or j (square root of -1); exp or e (base of natural logarithms); Re or Im (real or imaginary part); log, In, sin, cos, etc.; abbreviations such as c.c. (complex conjugate); multiletter symbols (e.g. TL for transmission loss); subscripts of two or more letters identifiable as words or word-abbreviations (e.g., Apipe, fmax)

For more unusual functions, JSV follows Abramowitz and Stegun's book. More detail given in the GFA (see link above).

Unit symbols - These should be upright (e.g. kg, not $\mathrm{kg}$ ).

All authors are listed on the manuscript with correct affiliations, correct email address and are in correct order.

Keywords present.

Manuscript is not currently submitted to any other Journal.

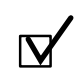

If submitting highlights please note that only six may be submitted and each one should be no longer than 85 characters in length.

Novelty of paper has been clearly stated in the Introduction.

References are presented as per GFA.

References not produced in English language to have English translation in brackets.

Figures and Tables and Equations are numbered in sequence correctly. (See GFA).

Nomenclature (if required) appears on second page of submission.

Acknowledgements should appear in a separate section just after the conclusions.

All abbreviations, in both the abstract and main body of document, are defined once only, the first time they appear in the text. (N.B. The Abstract is treated as an independent text, where references are given in full and abbreviations and symbols, if used, are properly defined.)

Figures - if there are multi-parts to a figures each part is labelled (a) (b) (c) etc. and the labels defined in the figure caption.

Figures - Colour can be used for the on-line version. Figures are reproduced in black and white in the printed journal and must therefore be readable in both colour and black \& white. (N.B. charges apply for production of colour figures in the printed journal)

Appendices - should appear before the list of references and labelled A, B, C, (please see GFA for further information regarding equations, figures and tables in the appendices.

Copyright - material reproduced from other publications (e.g. Tables, Figures), source is acknowledged.

Statement of Author contribution complete (see GFA) 
Abdel Darwish: Conceptualisation, Methodology, Software, Validation, Formal analysis, Investigation, Resources, Data Curation, Writing - Original Draft, Writing - Review \& Editing, Visualisation and Project administration.

Benjamin Halkon: Conceptualisation, Methodology, Software, Validation, Formal analysis, Investigation, Resources, Data Curation, Writing - Original Draft, Writing - Review \& Editing, Visualisation, Supervision, Project administration and Funding Acquisition.

Steve Rothberg: Validation, Formal analysis, Investigation, Writing - Review \& Editing.

Sebastian Oberst: Methodology, Validation, Resources, Writing - Review \& Editing, Supervision and Funding Acquisition.

Robert Fitch: Conceptualisation, Resources, Validation, Writing - Review \& Editing, Supervision and Funding Acquisition. 


\section{Declaration of interests}

$\bigotimes$ The authors declare that they have no known competing financial interests or personal relationships that could have appeared to influence the work reported in this paper.

$\square$ The authors declare the following financial interests/personal relationships which may be considered as potential competing interests:

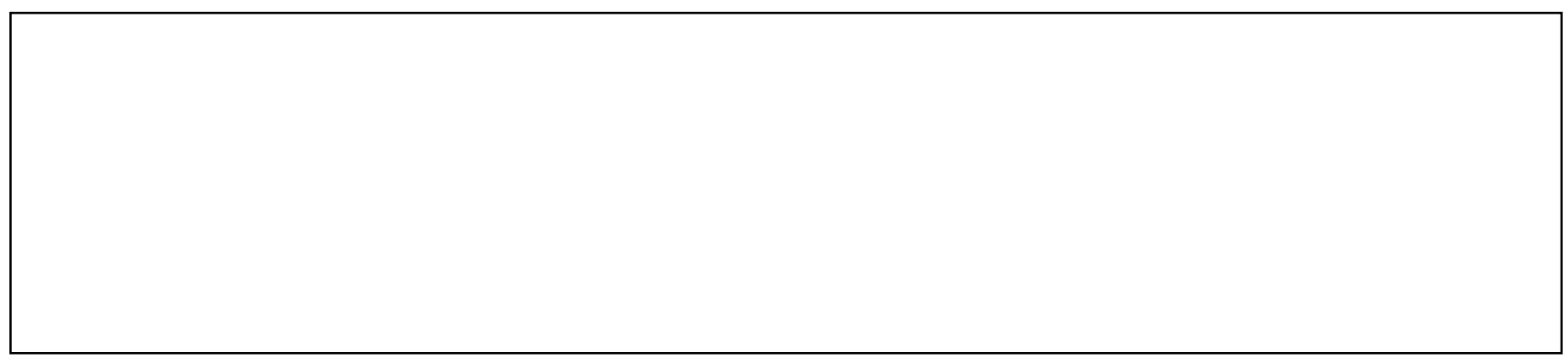

